\title{
Algorithms for crystallographic groups
}

\author{
Bettina Eick and Bernd Souvignier
}

April 29, 2005

\begin{abstract}
This article contains a survey on the algorithms for space groups and crystallographic groups available in the computer algebra system Gap [9] and in the software packages Carat [25] and Cryst [5].
\end{abstract}

\section{Introduction}

There are various problems on crystallographic groups which lead to interesting computational problems. Perhaps the most prominent problem arises from Bieberbach's famous theorem [1] stating that up to isomorphism there are only finitely many space groups in a given dimension. The problem of constructing the space groups in a given dimension has turned out to be a challenging computational problem.

Computational group theory is a branch of group theory which addresses such computational problems. Its aim is to design effective algorithms which can be used to compute with groups and to provide implementations which allow to solve interesting problems.

The computer algebra system Gap [9] provides a large variety of algorithmic methods to compute with groups of various types and it also allows to compute with crystallographic groups. It contains some special packages for this purpose: the CrystGap package [5] with some special algorithms for crystallographic groups and also the CrystCat package [6] with a database of space groups in small dimensions. Further, there is the software package Carat [25] which provides various methods especially designed for crystallographic groups. For the later package there is an interface [8] to the Gap system, so that all of these packages can be used from within Gap.

This paper is a survey on the methods made available by these software packages. It includes outlines on the methods and it gives some sample applications of the methods.

Various information about space groups has been listed in books and large tables, the most important source for 3-dimensional space groups being the International Tables [10]. However, the approach of tabulating results in books has its limitations: this is illustrated, for example, by the classification [2] of the space groups of dimension 4 , as only a small fraction of the information given in the International Tables could be provided in this case.

The data tabulated in such books can also be accessed very easily using Gap and Carat and, additionally, the software packages allow to compute more specific information for the considered groups beyond the information available in the tables. The power of the computational approach has, for example, been demonstrated by the complete enumeration of space groups up to dimension 6 in [26].

\section{Preliminaries}

In this section we describe the basic terminology used throughout this paper.

\subsection{Affine and Euclidian groups}

Let $\mathbb{R}^{n}$ be the $n$-dimensional real vector space consisting of column vectors

$$
\mathbb{R}^{n}=\left\{\left(\begin{array}{c}
x_{1} \\
\vdots \\
x_{n}
\end{array}\right) \mid x_{1}, \ldots, x_{n} \in \mathbb{R}\right\} .
$$

1 Definition: An affine mapping of $\mathbb{R}^{n}$ is a mapping of the form $v \mapsto g v+t$ where $g \in \operatorname{GL}(n, \mathbb{R})$ is an invertible matrix over $\mathbb{R}^{n}$ and $t \in \mathbb{R}^{n}$ is a vector. We denote this mapping by the Seitz notation $\{g \mid t\}$ and call $g$ the linear part and $t$ the translation part of $\{g \mid t\}$. 
Every affine mapping $\{g \mid t\}$ can be represented by a matrix

$$
\left(\begin{array}{l|l}
g & t \\
\hline 0 & 1
\end{array}\right) \in \mathrm{GL}(n+1, \mathbb{R}) .
$$

Using this representation, the application of an affine mapping $\{g \mid t\}$ to a vector $v \in \mathbb{R}^{n}$ now amounts to a multiplication of the matrix corresponding to $\{g \mid t\}$ with the vector $v$ augmented by an additional component 1 :

$$
\left(\begin{array}{l|l}
g & t \\
\hline 0 & 1
\end{array}\right) \cdot\left(\begin{array}{l}
v \\
1
\end{array}\right)=\left(\begin{array}{c}
g v+t \\
1
\end{array}\right)
$$

We often identify an affine mapping with its matrix representation as this simplifies notation.

2 Remark: Using the matrix representation of affine mappings one can readily observe that products and inverses of affine mappings are determined as follows

$$
\begin{aligned}
\{g \mid t\} \cdot\{h \mid s\} & =\{g h \mid t+g s\} \\
\{g \mid t\}^{-1} & =\left\{g^{-1} \mid-g^{-1} t\right\} .
\end{aligned}
$$

Thus the set of all affine mappings forms a group. This initiates the following definition.

3 Definition: We call $\operatorname{Aff}(n)$ the affine group of degree $n$ and $T(n)$ the translation subgroup of $\operatorname{Aff}(n)$ where

$$
\begin{aligned}
\operatorname{Aff}(n) & :=\left\{\{g \mid t\} \mid g \in \operatorname{GL}(n, \mathbb{R}), t \in \mathbb{R}^{n}\right\} \text { and } \\
T(n) & :=\left\{\{\mathbf{1} \mid t\} \mid t \in \mathbb{R}^{n}\right\} .
\end{aligned}
$$

Since conjugation of $\{\mathbf{1} \mid t\}$ by $\{h \mid s\}$ is given by $\left\{h^{-1} \mid-h^{-1} s\right\} \cdot\{\mathbf{1} \mid t\} \cdot\{h \mid s\}=\left\{\mathbf{1} \mid h^{-1} t\right\}$, it follows that $T(n)$ is invariant under conjugation with elements from $\operatorname{Aff}(n)$ and thus forms an invariant or normal subgroup of $\operatorname{Aff}(n)$.

4 Definition: Let $g^{T}$ be the transposed matrix for $g \in \mathrm{GL}(n, \mathbb{R})$. We call $\mathrm{E}(n)$ the Euclidian group of degree $n$ where

$$
\mathrm{E}(n):=\left\{\{g \mid t\} \in \operatorname{Aff}(n) \mid g^{T} g=\mathbf{1}\right\}
$$

Thus the Euclidian group is the set of those affine mappings whose linear part is contained in the orthogonal group $O(n)=\left\{g \in \mathrm{GL}(n, \mathbb{R}) \mid g^{T} g=1\right\}$. As $O(n)$ is a subgroup of $\mathrm{GL}(n, \mathbb{R})$, it follows readily that $\mathrm{E}(n)$ is a subgroup of $\operatorname{Aff}(n)$. Note that $T(n) \leq \mathrm{E}(n)$ by definition.

\subsection{Space groups}

For every $G \leq \mathrm{E}(n)$ it follows that $T=T(n) \cap G$ is a normal subgroup of $G$ which we call its translation subgroup and the factor group $G / T$ is called the point group of $G$.

We say that a subgroup $L$ of $\mathbb{R}^{n}$ is a lattice if $L=\mathbb{Z} b_{1}+\ldots+\mathbb{Z} b_{m}$ for $\mathbb{Z}$-linearly independent vectors $\left(b_{1}, \ldots, b_{m}\right)$. The vectors $\left(b_{1}, \ldots, b_{m}\right)$ are called a lattice basis for $L$ and the number $m$ of basis vectors is called the rank of $L$. We say that $L$ is a full lattice if $\left(b_{1}, \ldots, b_{m}\right)$ is a basis for $\mathbb{R}^{n}$ or, equivalently, if its rank equals $n$.

The translation subgroup $T$ of a subgroup $G$ of $\mathrm{E}(n)$ can be identified with a subgroup of $\mathbb{R}^{n}$ via $T \rightarrow \mathbb{R}^{n}:\{1 \mid t\} \mapsto t$. This is used in the following definition.

5 Definition: Let $G \leq \mathrm{E}(n)$ with translation subgroup $T=T(n) \cap G$. Then $G$ is called a crystallographic space group or space group for short if $T$ is a full lattice in $\mathbb{R}^{n}$. In this case $T$ is also called translation lattice of $G$.

Let $G$ be a space group. As its translation subgroup $T$ is a normal subgroup of $G$, we can consider the action of its point group $K$ on $T$. Fixing a lattice basis for $T$, this action is described by matrices in $\operatorname{GL}(n, \mathbb{Z})$ and hence we obtain an integral representation of $K$. Since only the identity element of $K$ acts as the identity matrix in this representation, we obtain an embedding of $K$ into $\operatorname{GL}(n, \mathbb{Z})$. We often identify the point group $K$ with this corresponding subgroup of $\mathrm{GL}(n, \mathbb{Z})$.

6 Lemma: The point group of a space group is a finite subgroup of $O(n)$.

Proof: Let $T$ be the translation subgroup of the space group $G$ and let $\left(b_{1}, \ldots, b_{n}\right)$ be a lattice basis of $T$. There are only finitely many vectors in $T$ to which a basis vector $b_{i}$ can be mapped under an element of the point group $K$, since the image has to be a vector of the same length as $b_{i}$. But this leaves only finitely many possibilities for the columns of an element of the point group $K$.

A lattice basis for the translation subgroup $T$ of a space group $G$ is also a basis of $\mathbb{R}^{n}$. Thus we can represent $G$ by affine matrices with respect to this lattice basis. Then the translations in $T$ coincide with $\left\{\{1 \mid t\} \mid t \in \mathbb{Z}^{n}\right\}$. This is called the standard 
form of the space group $G$. We investigate this standard form in the following.

Let $G$ be a space group in standard form. Then the point group of $G$ can be identified with the image $K$ of $G \rightarrow \mathrm{GL}(n, \mathbb{Z}):\{g \mid t\} \mapsto g$ and the action of $K$ on $\mathbb{Z}^{n}$ coincides with the action of $G / T$ on $T$.

7 Definition: Let $G$ be a space group with point group $K$.

(a) For every $g \in K$ choose some $t_{g} \in \mathbb{R}^{n}$ with $\left\{g \mid t_{g}\right\} \in G$. Then the map $\tau: K \rightarrow \mathbb{R}^{n}: g \mapsto$ $t_{g}$ is called a vector system. The set of images $\left\{t_{g} \mid g \in K\right\}$ is called a system of nonprimitive translations.

(b) Space groups containing a subgroup isomorphic to their full point group are called symmorphic space groups. This is the case if and only if the image of $\tau$ lies in $\mathbb{Z}^{n}$.

Multiplication of space group elements shows that a vector system satisfies the cocycle condition:

$$
\tau(g h) \equiv \tau(g)+g \cdot \tau(h) \bmod \mathbb{Z}^{n} \text { for all } g, h \in K .
$$

In summary, a space group $G$ in standard form is characterized by its point group $K \leq \operatorname{GL}(n, \mathbb{Z})$ and a vector system $\tau$. We therefore obtain that it is of the form

$$
G=\left\{\left(\begin{array}{c|c}
g & t_{g}+t \\
\hline 0 & 1
\end{array}\right) \mid g \in K, t \in \mathbb{Z}^{n}\right\} .
$$

\subsection{Bravais manifolds}

For a lattice $T$ with basis $B=\left(b_{1}, \ldots, b_{n}\right)$, the matrix $F$ with the inner products $F_{i j}:=b_{i} \cdot b_{j}$ as entries is called the metric tensor of $T$ (with respect to $B$ ). If $G$ is a space group with translation lattice $T$ and point group $K$, then for every $g \in K$ it follows that the basis $B$ transforms into $\left(g b_{1}, \ldots, g b_{n}\right)$ and the metric tensor $F$ of $T$ transforms into $g^{T} F g$. As $K$ consists of orthogonal transformations only, the inner product and thus the metric tensor of the translation lattice $T$ is fixed under the action of $K$. Thus it follows that $g^{T} F g=F$ holds for all $g \in K$.

8 Definition: The Bravais manifold $\mathcal{F}(K)$ of an integral matrix group $K \leq \mathrm{GL}(n, \mathbb{Z})$ is the space of all metric tensors invariant under $K$ :

$$
\mathcal{F}(K):=\left\{F \in \mathbb{R}_{\text {sym }}^{n \times n} \mid g^{T} F g=F \text { for all } g \in K\right\}
$$

It is straightforward to observe that $\mathcal{F}(K)$ is an $\mathbb{R}$-vector space. Moreover, for a finite group $K$ the Bravais manifold $\mathcal{F}(K)$ always contains a positive definite metric tensor; that is, a tensor $F$ with $v^{T} F v>0$ for all $v \neq 0$. For example, the average tensor $F_{0}:=\sum_{g \in K} g^{T} g$ has this property.

The dimension of the Bravais manifold of a finite group $K \leq \mathrm{GL}(n, \mathbb{Z})$ can be read off the irreducible characters involved in the natural character $\rho: K \rightarrow \mathbb{C}: g \mapsto \operatorname{trace}(g)$, see [24]. Suppose that the decomposition of $\rho$ into (complex) irreducible characters of $K$ is

$$
\rho=\sum_{i=1}^{r} a_{i} \chi_{i}+\sum_{j=1}^{s} b_{j} \psi_{j}+\sum_{k=1}^{u} c_{k}\left(\theta_{k}+\overline{\theta_{k}}\right),
$$

where $\chi_{i}$ are the characters of real representations, $\psi_{j}$ are the characters with real values and representations not realizable over $\mathbb{R}$ and $\theta_{k}$ are the remaining characters, coming in pairs with their complex conjugates. Then the dimension of the Bravais manifold is given by

$\operatorname{dim}_{\mathbb{R}}(\mathcal{F}(K))=\sum_{i=1}^{r} \frac{a_{i}^{2}+a_{i}}{2}+\sum_{j=1}^{s} \frac{b_{j}^{2}-b_{j}}{2}+\sum_{k=1}^{u} c_{k}^{2}$.

In particular, a group admits (up to scalings) a unique invariant metric tensor if and only if the natural character $\rho$ is either irreducible, is the sum of two complex characters or is of the form $2 \psi$ for a real character $\psi$ not realizable over $\mathbb{R}$.

9 Definition: Let $\mathcal{F} \subseteq \mathbb{R}_{\text {sym }}^{n \times n}$ be a set of metric tensors and $K \leq \operatorname{GL}(n, \mathbb{Z})$ an integral matrix group.

(a) We define the Bravais group $\mathcal{B}(\mathcal{F})$ of $\mathcal{F}$ as $\{g \in$ $\operatorname{GL}(n, \mathbb{Z}) \mid g^{T} F g=F$ for all $\left.F \in \mathcal{F}\right\}$.

(b) The Bravais group $\mathcal{B}(\mathcal{F}(K))$ of the Bravais manifold $\mathcal{F}(K)$ of $K$ is called the Bravais group of $K$.

If $\mathcal{F}$ contains a positive definite tensor, then $\mathcal{B}(\mathcal{F})$ is necessarily finite, since the images of a lattice basis of $\mathbb{Z}^{n}$ lie in the finite set of vectors of the same length as the basis vectors.

The Bravais group $\mathcal{B}(K)$ is the full group of symmetries of the lattice on which $K$ acts. A point group which coincides with its Bravais group is also called a Bravais group or a holohedry.

10 Definition: We call a metric tensor $F \in \mathcal{F}$ a generic form of $\mathcal{F}$ if $\mathcal{B}(F)=\mathcal{B}(\mathcal{F})$ holds. 
We note that $F$ is a generic form in the Bravais manifold of $\mathcal{F}:=\mathcal{F}(\mathcal{B}(F)$ ). It is shown in [20] that almost all positive definite metric tensors in a Bravais manifold are generic forms. More precisely, the positive definite metric tensors which are not generic lie on countably many hyperplanes in the Bravais manifold and therefore form a subset of measure 0 .

Example: The full group $2 \mathrm{~mm}$ of symmetries of a rectangular translation lattice fixes the 2-dimensional space of metric tensors given by

$$
\mathcal{F}=\left\{\left(\begin{array}{ll}
a & 0 \\
0 & b
\end{array}\right) \mid a, b \in \mathbb{R}\right\} .
$$

In $\mathcal{F}$, all metric tensors with $a \neq b$ are generic forms, whereas the tensors with $a=b$ are not generic, since their Bravais group is the larger group $4 m m$.

11 Definition: For a lattice $T$ with metric tensor $F$, the group $\mathcal{B}(F)$ of all symmetries of $T$ is called the Bravais group of $T$.

We say that a lattice $T^{\prime}$ lies in the same Bravais class as $T$ if the Bravais groups of $T$ and $T^{\prime}$ coincide up to a transformation of lattice bases. But this is equivalent to saying that for a suitably chosen basis the metric tensor of $T^{\prime}$ is also a generic form in $\mathcal{F}$. The generic forms in a Bravais manifold therefore correspond to lattices belonging to the same Bravais class and the Bravais manifold itself represents this Bravais class.

\section{General group theoretic al- gorithms}

This section gives a brief overview on general methods from computational group theory as far as they are relevant for computations with space groups. These methods are implemented in computer algebra systems like Gap [9] and Magma [18]. We refer to [14] for background and more details.

Space groups are often described by their point groups and a vector system exhibiting how the point group and the translations are glued together. The point groups can be considered as finite subgroups of $\mathrm{GL}(n, \mathbb{Z})$ and there is a whole range of methods from computational group theory which apply to such groups. For different kinds of questions different ways to represent a finite group are favorable. The most important being:

- permutation groups acting on a set of points;

- matrix groups acting on a vector space;

- finitely presented groups given by generators and relators.

There are various methods available to compute with groups of these types, usually the methods depend heavily on the considered representation. It is also important to be able to switch between these types of representation and computational group theory provides various methods for this purpose.

\subsection{Orbits and Stabilizers}

We say that a group $G$ acts on a set $\Omega$ if for every $g \in G$ and $\omega \in \Omega$ there is a unique element $g(\omega) \in \Omega$ to which $\omega$ is mapped under $g$ such that $g(h(\omega))=(g h)(\omega)$ for all $g, h \in G$ and $\omega \in \Omega$ and $1(\omega)=\omega$ for all $\omega \in \Omega$. Typical examples are:

- the natural action of a space group on the points of $\mathbb{R}^{n}$;

- the natural action of a point group on the points of $\mathbb{Z}^{n}$;

- the conjugation action of a group $G$ on its elements given by $g(h):=g h g^{-1}$;

- the conjugation action of a group $G$ on its subgroups given by $g(U):=g \cdot U \cdot g^{-1}$.

For a given point $\omega \in \Omega$, the set

$$
G(\omega):=\{g(\omega) \mid g \in G\}
$$

of points to which $\omega$ is mapped is called the orbit of $\omega$ under $G$ and the subgroup

$$
G_{\omega}=\operatorname{Stab}_{G}(\omega):=\{g \in G \mid g(\omega)=\omega\}
$$

of group elements fixing $\omega$ is called the stabilizer of $\omega$ in $G$. In the case of a space group acting on the points of $\mathbb{R}^{n}$, the stabilizer of a point is often called the site-symmetry group.

The computation of orbits and stabilizers is one of the crucial tasks in computational group theory and there are highly efficient methods available if the considered orbits are finite. If the orbits in 
question are infinite, then the determination of orbits and stabilizers is generally not possible by general methods.

We note that computing generators for the stabilizer of a point requires more work than computing the orbit, but it is still quite effective. If the stabilizer of a point is determined, then the orbit can be read off from the cosets of $G_{\omega}$ in $G$ : For $g \in G$, the coset $G_{\omega} g:=\left\{h g \mid h \in G_{\omega}\right\}$ consists of all elements of $G$ mapping $\omega$ to the same orbit point $g(\omega)$ as $g$.

\section{$3.2 \quad$ Permutation groups}

Permutation groups allow particularly efficient algorithms to compute with this type of group. Thus it is often useful to convert a finite group into a permutation group for computational purposes. We refer to Chapter 4 of [14] for a detailed discussion of permutation group methods.

For permutation groups of moderate size, as they are relevant for the study of crystallographic groups, usually every desired information can be computed explicitly, including the full lattice of subgroups of a group, the character table or the automorphism group.

There are effective methods available to transform a finite matrix group into a permutation group. The most obvious approach is to use the action of the matrix group on the orbits of the basis vectors of the underlying vector space. Using this transformation, it is also straightforward to translate a permutation back into its original matrix.

\subsection{Matrix Representations}

Point groups of space groups are usually explicitly given as finite matrix groups in $\operatorname{GL}(n, \mathbb{Z})$. Again, there is a large variety of methods available to compute with such groups. An often used approach is to determine a permutation representation for them and then use permutation group methods. Based on this approach, one can compute all kinds of information for point groups of moderate degree.

A second issue concerned with matrix representations comes up in the computation of invariant sublattices of the translation lattice. Here, the irreducible constituents of a matrix subgroup of $\operatorname{GL}\left(n, \mathbb{F}_{p}\right)$ for a prime $p$ are required. These constituents can be determined by the so-called Meataxe algorithm, see Section 7.4 of [14].

\subsection{Finitely presented groups}

A finite presentation $\left\langle g_{1}, \ldots, g_{m} \mid r_{1}, \ldots, r_{s}\right\rangle$ consists of a set of abstract generators $g_{1}, \ldots, g_{m}$ and a set of relators $r_{1}, \ldots, r_{s}$ where every relator is a word in the generators $r_{i}=r_{i}\left(g_{1}, \ldots, g_{m}\right)$. The group $G$ defined by a such a finite presentation can be thought of as the largest group generated by generators $\bar{g}_{1}, \ldots, \bar{g}_{m}$ satisfying the relators in the form $r_{i}\left(\bar{g}_{1}, \ldots, \bar{g}_{m}\right)=1$ for all $i$.

Example: Let $D_{n}$ be the dihedral group of order $2 n$. Then $D_{n}$ is generated by an $n$-fold rotation $\bar{x}$ and a reflection $\bar{y}$. Let $G=\left\langle x, y \mid x^{n}, y^{2}, y^{-1} x y x\right\rangle$. Then $\bar{x}$ and $\bar{y}$ satisfy the relators of $G$. It is also not difficult to observe that $D_{n}$ is a largest group with this property. Hence $D_{n} \cong G$ and $D_{n}$ is defined by the finite presentation of $G$.

We note that several group theoretic problems are in general undecidable in finitely presented groups. Hence computations with this type are limited. However, there are also some problems in computational group theory which require a finite presentation to solve them. For example, representing a group by abstract generators and relators is required in the computation of the space groups with given point group by the Zassenhaus algorithm, see Section 4.5.

If a finitely presented group is known to be finite, then the coset enumeration method can be used to construct an isomorphism into a permutation group. See Chapter 5 of [14]. As point groups of space groups are always finite, this method can be applied to them.

There are special methods to compute with special types of finitely presented groups. An example of such a special type are the so-called polycyclicly presented groups. A group $G$ is polycyclic if it has a chain $G=N_{0} \unrhd N_{1} \ldots \unrhd N_{r}=\{1\}$ of subnormal subgroups such that the successive quotients $N_{i} / N_{i+1}$ are cyclic groups. Every polycyclic group has a so-called polycyclic presentation and this type of presentation allows effective computations, see Chapter 8 of [14].

We note that a space group is polycyclic if and only if its point group is polycyclic. In particular, all space groups of degree at most 3 are polycyclic and in degree 4 only the space groups with point group containing the alternating group $A_{5}$ are not polycyclic. 


\subsection{Character tables}

Representations and character tables of groups are an often used tool from group theory which is relevant in crystallography and solid state physics. For example, splitting tensor products of characters into irreducible characters is required for the determination of infrared or Raman spectra.

Therefore it is desirable to have the character table of a group available and to be able to compute with the characters. The Dixon-Schneider algorithm, see Section 7.7 of [14], provides a method to determine the character table of a finite group. This method is powerful enough to be able to deal with all point groups in moderate degrees.

Another example for an application in chemistry of the character theory as provided by the Gap system is given in [28].

\subsection{Summary}

The point groups of space groups or the quotient groups of a space group by a subgroup of the translation lattice of finite index are often of central interest in crystallography. For moderate dimensions, these groups can be regarded as small from the point of view of computational group theory. For example, in dimension 3 the largest point group is the symmetry group of the cube of order 48. Even in dimension 6 the symmetry group of the root lattice $E_{6}$ of order 103680 is still easily manageable by standard tools from computational group theory.

One may therefore say, that for the finite groups arising in crystallography, a lot of information can be computed explicitly and fast. This includes (but is not restricted to):

- deciding conjugacy of elements or subgroups;

- deciding inclusion of elements or subgroups in other subgroups;

- computing the lattice of subgroups of a group;

- expressing an element as products of given generators;

- computing generators and relators;

- computing character tables;

- computing automorphism groups.

\section{Fundamental algorithms}

In this section we discuss some important and wellknown specific algorithms for space groups as implemented in Carat [25] or CrystGap [6]. More information about the methods and their theoretical background can be found in [22].

\subsection{Bravais groups and isometries}

Bravais classes of lattices play an important role in the classification of space groups. In turn, the computation of the full group of automorphisms of a lattice is fundamental in this area.

\subsubsection{Automorphism groups}

Let $L$ be a lattice with metric tensor $F$ and denote $G=\operatorname{Aut}(L)$. Then $G$ can be determined from $L$ and $F$ with the following approach. Let $B=\left(b_{1}, \ldots, b_{n}\right)$ be a lattice basis of $L$. Then an automorphism $\varphi$ of $L$ maps the basis $B$ to a new basis

$$
C=\left(c_{1}, \ldots, c_{n}\right) \text { with } c_{i}=\varphi\left(b_{i}\right) .
$$

The inner products of the image vectors coincide with those of the original basis; that is, it follows that $c_{i}^{T} \cdot c_{j}=F_{i j}$. In particular, every vector $c_{i}$ is contained in the set

$$
M:=\left\{v \in L \mid v^{T} \cdot v \leq \max \left(F_{11}, \ldots, F_{n n}\right)\right\}
$$

of all vectors up to the maximal length of the basis vectors. As $F$ is a positive definite metric tensor, the set $M$ is finite and hence $G$ can be computed as a subgroup of the group of permutations of $M$.

This computation is accomplished in a backtrack search, using techniques analogous to those for permutation groups. The core idea is to construct the matrix of an automorphism $\varphi$ column by column: The first column is a vector $c_{1} \in M$ with $c_{1}^{T} \cdot c_{1}=F_{11}$. After choosing $c_{1}$, the candidates for the second column are restricted to those vectors in $M$ having the same length as $b_{2}$ and inner product $F_{12}$ with $c_{1}$. Some invariants are used in order to prune branches in the backtrack search as early as possible. We refer to [27] for a detailed account of the method.

\subsubsection{Bravais groups}

The computation of the automorphism group of a lattice can readily be extended to the computation 
of the Bravais group of a Bravais manifold. This is immediate if we think of the metric tensor $F$ as being a generic tensor in the Bravais manifold.

However, it is often more convenient to represent a Bravais manifold by a basis $\left(F_{1}, \ldots, F_{m}\right)$ of linearly independent metric tensors, the first of which is positive definite. In that case, the Bravais group is computed by the automorphism group algorithm with the only change that the inner products of the new basis $\left(c_{1}, \ldots, c_{n}\right)$ not only have to be correct with respect to $F_{1}$, but also those with respect to the other metric tensors. This yields further restrictions for the vectors that are allowed as columns of the matrix of an automorphism $\varphi$.

\subsubsection{Isometries}

The algorithm for computing the automorphism group of a lattice can easily be adapted in order to compute an isometry between two lattices $L$ and $L^{\prime}$ with metric tensors $F$ and $F^{\prime}$, respectively. An isometry maps a basis of $L$ to a basis of $L^{\prime}$ having inner products given by $F$ and thus the columns of the matrix of an isometry are short vectors in the lattice $L^{\prime}$ which fulfill the restrictions imposed by the metric tensor $F$.

In turn, the method to compute an isometry between two lattices can be extended to the computation of an isometry between Bravais manifolds. However, since there is in general no obvious choice for a generic lattice, one has to find a correspondence between the metric tensors in the two Bravais manifolds. An efficient solution to this problem is provided by the perfect forms and their directions which will be described in more detail in Section 4.2 about the computation of the integral normalizer.

\subsubsection{Sample applications}

The algorithms described are highly efficient and depend mainly on the number of short vectors in a lattice. In low dimensions these are very small. For example, the highly symmetric root lattice $E_{6}$ in dimension 6 has only 72 vectors of length 2 and generators for its automorphism group are found instantaneously in practice. Even for the 24-dimensional Leech lattice with 196560 vectors of minimal length 4 , generators for the full automorphism group of order $2^{22} \cdot 3^{9} \cdot 5^{4} \cdot 7^{2} \cdot 11 \cdot 13 \cdot 23 \approx 8.3 \cdot 10^{18}$ are computed in few minutes.

\subsection{Integral normalizers}

In many computations with space groups, in addition to the space group itself also the integral normalizer of its point group is required. In general, the normalizer $N_{G}(U)$ in $G$ of a subgroup $U \leq G$ is defined as

$$
N_{G}(U):=\left\{g \in G \mid g U g^{-1}=U\right\}
$$

and is the largest subgroup of $G$ in which $U$ is a normal subgroup. In terms of orbits and stabilizers, the normalizer is the stabilizer of $U$ under the conjugation action of $G$ on its subgroups. In the context of space groups, the integral normalizer $N_{\mathrm{GL}(n, \mathbb{Z})}(K)$ of a point group $K$ can be interpreted as the geometric automorphism group of the situation considered, since it reflects the different possibilities of labeling the elements of $K$ such that their geometric functions remain the same.

In this section we discuss two different approaches for computing the integral normalizer of a point group. The first one is purely group theoretic and relies only on the fact that elements from the normalizer realize automorphisms of the abstract group. It was successfully used in the classification of space groups of dimension 4 in [2]. The second approach uses the fact that the integral normalizer acts on the Bravais manifold of a point group.

\subsubsection{Using group automorphisms}

Let $K \leq \mathrm{GL}(n, \mathbb{Z})$ be a finite group and let $A u t(K)$ denote its (abstract) automorphism group. The following remark yields a connection between $A u t(K)$ and $N_{\mathrm{GL}(n, \mathbb{Z})}(K)$.

12 Remark: The conjugation action of $g \in$ $N_{\mathrm{GL}(n, \mathbb{Z})}(K)$ defines an automorphism $\sigma_{g}: K \rightarrow$ $K: h \mapsto g h g^{-1}$ of $K$ and the mapping $\sigma$ : $N_{\mathrm{GL}(n, \mathbb{Z})}(K) \rightarrow \operatorname{Aut}(K): g \mapsto \sigma_{g}$ is a homomorphism.

As $K$ is a finite group, its automorphism group $\operatorname{Aut}(K)$ can be computed readily and it is again a finite group. It now remains to obtain $N_{\mathrm{GL}(n, \mathbb{Z})}(K)$ from $\operatorname{Aut}(K)$. For this purpose we investigate for a given $\varphi \in A u t(K)$ whether $\varphi$ has a preimage under $\sigma$ and, if so, then we determine all such preimages.

Let $K=\left\langle h_{1}, \ldots, h_{r}\right\rangle$ and let $h_{i}^{\prime}=\varphi\left(h_{i}\right)$ for all $i$. We want to test whether there exists an integral matrix $g \in \mathrm{GL}(n, \mathbb{Z})$ with $g h_{i} g^{-1}=h_{i}^{\prime}$ for all $1 \leq i \leq r$. Every such $g$ arises as solution of the 
homogeneous system of linear equations in the $n^{2}$ entries of $X$ for the matrix equations

$$
X h_{i}-h_{i}^{\prime} X=0 \text { for } 1 \leq i \leq r .
$$

When solving this system, the following outcomes have to be considered:

(i) The system has an integral solution $g$ with $\operatorname{det}(g)= \pm 1$. Then $g \in N_{\mathrm{GL}(n, \mathbb{Z})}(K)$ follows.

(ii) The system has a rational solution $g$, but no integral solution with $\operatorname{det}(g)= \pm 1$. Then $\varphi \notin$ $\operatorname{Im}(\sigma)$ follows.

(iii) The system has no solution. Then $\varphi \notin \operatorname{Im}(\sigma)$ follows.

The dimension of the solution space is either 0 or equals the dimension of the centralizing algebra $C_{\mathbb{Q}}(K):=\left\{X \in \mathbb{Q}^{n \times n} \mid X h=h X\right.$ for all $\left.h \in K\right\}$. If its dimension is 0 , then we are in case (iii) and there is nothing to do. If its dimension is 1 , then cases (i) and (ii) can be distinguished. However, if its dimension is larger than 1, then it might be difficult to decide whether we are in case (i) or (ii) and this is the general difficulty inherent in this approach.

\subsubsection{Using perfect forms}

The second approach to computing integral normalizers uses the Bravais manifold. The first important observation is that the integral normalizer of an arbitrary point group $K$ is a subgroup of the normalizer of its Bravais group $\mathcal{B}(K)$.

13 Lemma: Let $K \leq \mathrm{GL}(n, \mathbb{Z})$ be finite and let $B:=\mathcal{B}(K)$ be its Bravais group. Then $N_{\mathrm{GL}(n, \mathbb{Z})}(K) \leq N_{\mathrm{GL}(n, \mathbb{Z})}(B)$.

Proof: Let $\mathcal{F}:=\mathcal{F}(K)$ be the Bravais manifold of $K$, then $\mathcal{F}(B)=\mathcal{F}$, since $B$ is the Bravais group of $K$. For $g \in N_{\mathrm{GL}(n, \mathbb{Z})}(K)$ and $F \in$ $\mathcal{F}$ we have $g^{T} F g \in \mathcal{F}$, since for any $h \in K$ one has $g h=h^{\prime} g$ for some $h^{\prime} \in K$ and thus $h^{T}\left(g^{T} F g\right) h=g^{T}\left(h^{\prime T} F h^{\prime}\right) g=g^{T} F g$. But this implies that for $b \in B$ and $g \in N_{\mathrm{GL}(n, \mathbb{Z})}(K)$ one has $\left(g b g^{-1}\right)^{T} F\left(g b g^{-1}\right)=g^{-T} b^{T}\left(g^{T} F g\right) b g^{-1}=$ $g^{-T}\left(g^{T} F g\right) g^{-1}=F$, since $b$ fixes $g^{T} F g \in \mathcal{F}$.

This lemma suggests the following reduction to Bravais groups: Let $K$ be a point group and let $N:=N_{\mathrm{GL}(n, \mathbb{Z})}(\mathcal{B}(K))$ be the integral normalizer of its Bravais group. Then $N_{\mathrm{GL}(n, \mathbb{Z})}(K) \leq N$ and thus $N_{\mathrm{GL}(n, \mathbb{Z})}(K)=\operatorname{Stab}_{N}(K)$ follows where $N$ acts on subgroups of $G L(n, \mathbb{Z})$ by conjugation. The orbit of $K$ under $N$ is finite and thus the corresponding stabilizer can be computed with methods from computational group theory, see Section 3.1. In fact, the orbit of $K$ is usually quite small, thus the reduction step is usually very efficient.

It remains to find the integral normalizer of a Bravais group. For that, the notion of perfect forms is important. Let $F$ be a positive definite metric tensor and let $\mu(F):=\min \left(v^{T} F v \mid v \in \mathbb{Z}^{n}\right\}$ be the minimum of $F$. Then the set $M(F):=\left\{v \in \mathbb{Z}^{n} \mid\right.$ $\left.v^{T} F v=\mu(F)\right\}$ is called the set of minimal vectors of $F$.

14 Definition: A metric tensor $F$ is called a perfect form if the set $D:=\left\{x \cdot x^{T} \mid x \in M(F)\right\}$ contains a basis for the vector space $R_{s y m}^{n \times n}$ of symmetric metric tensors.

We note that perfect forms yield good candidates for lattices with high packing density and refer the reader to [19] for a thorough discussion of the concept of perfect forms.

It was shown by Voronoi in [31] that there are only finitely many perfect forms of degree $n$ up to the action of $\operatorname{GL}(n, \mathbb{Z})$; that is, up to a base change in $\mathbb{Z}^{n}$. Voronoi also introduced an algorithm to compute the perfect forms. His result was generalized to the situation of metric tensors invariant under a finite group $G$ in [15] and [23]. The crucial idea is to consider the Bravais manifold $\mathcal{F}(G)$ together with the Bravais manifold $\mathcal{F}\left(G^{T}\right)$ for the group of transposed matrices. Note that the map

$$
\pi: \mathbb{R}_{s y m}^{n \times n} \rightarrow \mathcal{F}\left(G^{T}\right): F \mapsto \frac{1}{|G|} \sum_{g \in G} g F g^{T}
$$

is a projection onto $\mathcal{F}\left(G^{T}\right)$. Keeping the above notation for perfect forms, one says that a metric tensor $F \in \mathcal{F}(G)$ is $G$-perfect if $\pi(D)$ contains a basis of $\mathcal{F}\left(G^{T}\right)$.

15 Theorem: (Jacquet-Chiffelle, Opgenorth) There exist only finitely many $G$-perfect forms up to the action of the normalizer $N_{\mathrm{GL}(n, \mathbb{Z})}(G)$.

The original result of Voronoi is the special case where $G$ is the trivial group (which has $\operatorname{GL}(n, \mathbb{Z})$ as its normalizer). 
As in the case of perfect forms without a group acting, $G$-perfect forms can be constructed efficiently. One obtains a partition of the cone of positive definite $G$-invariant forms into cells and the hyperplanes separating the cells of two perfect forms are of the form

$$
H(Y)=\left\{X \in \mathbb{R}_{\text {sym }}^{n \times n} \mid \operatorname{trace}(X \cdot Y)=0\right\} .
$$

The forms $Y$ yielding the boundaries of the cell of a given perfect form $F$ are called the directions of $F$ and characterize the neighborship relations of the cells. It is shown in [23] that the set of directions contains a basis of $\mathcal{F}\left(G^{T}\right)$. Since the action of the integral normalizer on $\mathcal{F}(G)$ preserves the neighborship relations between the cells of $G$ perfect forms, a perfect form is always transformed together with its directions. As there are only finitely many $G$-perfect forms up to the action of the normalizer this gives a way to find an almost canonical basis for the Bravais manifold.

The algorithm to find generators for the normalizer then uses the following approach: After computing an initial $G$-perfect form, find all its neighbors and check whether an isometry between the perfect forms can be found that also transforms the directions into the directions of the other form. This can be facilitated by the methods of Section 4.1. Iterating this process ultimately yields generators for the integral normalizer of $G$.

\section{$4.3 \mathbb{Z}$-equivalence of point groups}

The approach for computing the integral normalizer via $G$-perfect forms and their directions as in Section 4.2 yields a method to decide whether two point groups are $\mathbb{Z}$-equivalent; that is, whether they are conjugate by a matrix in $\mathrm{GL}(n, \mathbb{Z})$.

We first consider the case of $\mathbb{Z}$-equivalence for Bravais groups. If two Bravais groups $B_{1}, B_{2}$ are conjugate by a transformation $g \in \mathrm{GL}(n, \mathbb{Z})$, then this transformation induces isometries between the metric tensors in the Bravais manifolds of $B_{1}$ and $B_{2}$. In particular, a $B_{1}$-perfect form has to be mapped to a $B_{2}$-perfect form by an isometry which also maps the directions of the first form to the directions of the second form. Finding such an isometry is accomplished by an adapted version of the algorithm to compute isometries between lattices described in Section 4.1.

Now let $K_{1}, K_{2} \leq \mathrm{GL}(n, \mathbb{Z})$ and suppose we want to decide whether these groups are $\mathbb{Z}$-equivalent.
First, one checks whether their Bravais groups are $\mathbb{Z}$-equivalent. If not, the groups can not be $\mathbb{Z}$ equivalent, otherwise one may assume that $K_{1}$ and $K_{2}$ are subgroups of the same Bravais group $\mathcal{B}\left(K_{1}\right)=\mathcal{B}\left(K_{2}\right)$. In that case they are $\mathbb{Z}$-equivalent if and only if they are conjugate under an element of the normalizer $N:=N_{\mathrm{GL}(n, \mathbb{Z})}\left(\mathcal{B}\left(K_{1}\right)\right)$. This is decided by computing the orbit of $K_{1}$ under $N$ and checking whether $K_{2}$ is contained in this orbit.

\subsection{Invariant sublattices}

If $G$ is a space group, then its translation lattice $T$ is setwise invariant under the action of its point group $K$. In this section we discuss the computation of other full lattices $L \subseteq \mathbb{Q}^{n}$ which are setwise invariant under the action of a given finite subgroup $K$ of $\mathrm{GL}(n, \mathbb{Z})$. These lattices are called $K$-invariant lattices.

Obvious examples of $K$-invariant lattices are the scalings $a \mathbb{Z}^{n}$ for $a \in \mathbb{Q}$. Of special interest are the $K$-invariant sublattices of $\mathbb{Z}^{n}$ which are not contained in a proper scaling $m \mathbb{Z}^{n}$ for an integer $m>1$ and which are usually termed centerings.

Example: Let $T$ be the usual square lattice $\mathbb{Z}^{2}$ and let $K$ be the point group $4 \mathrm{~mm}$. Then a $K$ invariant sublattice of $T$ is spanned by the vectors

$$
\left(\begin{array}{l}
1 \\
1
\end{array}\right) \text { and }\left(\begin{array}{c}
1 \\
-1
\end{array}\right) \text {. }
$$

This is also known as the checkerboard lattice.

An arbitrary $K$-invariant lattice $L$ can always be scaled such that the scaling is contained in $\mathbb{Z}^{n}$. Thus, we will only consider $K$-invariant sublattices of $\mathbb{Z}^{n}$ in the sequel.

In order to find $K$-invariant sublattices, it is sufficient to find maximal $K$-invariant sublattices and to iterate the procedure. If a maximal $K$-invariant sublattice $L$ of $\mathbb{Z}^{n}$ is given, then the index of $L$ in $\mathbb{Z}^{n}$ is a power of a prime $p$. Thus $L$ contains the $K$-invariant lattice $p \mathbb{Z}^{n}$.

16 Lemma: Let $L$ be a maximal $K$-invariant lattice in $T:=\mathbb{Z}^{n}$ such that $p T \subseteq L$. Then $L / p T$ is a maximal $\mathbb{F}_{p} K$-submodule of the natural $\mathbb{F}_{p} K$-module $T / p T$ and $T / L$ is an irreducible $\mathbb{F}_{p} K$-module.

Proof: The condition that $L$ is a $K$-invariant lattice translates readily to the fact that $L / p T$ is a $K$ submodule of $T / p T$. As $L$ is a maximal $K$-invariant 
lattice, it follows that $L / p T$ is a maximal submodule of $T / p T$.

If a finite subgroup $K$ of $\mathrm{GL}(n, \mathbb{Z})$ is given, then the action of $K$ on the natural $\mathbb{F}_{p} K$-module $T / p T$ for $T=\mathbb{Z}^{n}$ can be read off by reducing the matrixelements of $K$ modulo $p$.

The so-called Meataxe, see Section 3.3, can be used to determine all kinds of submodules in a given $\mathbb{F}_{p} K$-module. In particular, all maximal submodules can be determined readily.

Thus we can effectively determine all $K$-invariant maximal sublattices in a given lattice $L$ which contain $p L$ for a given prime $p$.

Example: Let $K$ be the point group

$$
31 m=\left\langle g=\left(\begin{array}{ll}
0 & -1 \\
1 & -1
\end{array}\right), h=\left(\begin{array}{ll}
0 & 1 \\
1 & 0
\end{array}\right)\right\rangle
$$

and let $T=\mathbb{Z}^{2}$.

- Consider the prime 2. Then $T / 2 T$ is irreducible as an $\mathbb{F}_{2} K$-module and hence $2 T$ is the only maximal $K$-invariant sublattice of $T$ containing $2 T$.

- Consider the prime 3. Then $T / 3 T$ has exactly one proper $\mathbb{F}_{3} K$-invariant submodule which is generated by

$$
s:=\left(\begin{array}{c}
1 \\
-1
\end{array}\right)
$$

Thus there exists exactly one $K$-invariant maximal sublattice $L$ in $T$ with $3 T \leq L$ and this is given by $L=\langle s, 3 T\rangle=\left\langle\left(\begin{array}{c}1 \\ -1\end{array}\right),\left(\begin{array}{l}0 \\ 3\end{array}\right)\right\rangle$.

The rational normalizer

$$
\begin{aligned}
N & :=N_{\mathrm{GL}(n, \mathbb{Q})}(K) \\
& =\left\{g \in \mathrm{GL}(n, \mathbb{Q}) \mid g K g^{-1}=K\right\}
\end{aligned} .
$$

of the point group $K$ acts on the $K$-invariant lattices, since for a $K$-invariant sublattice $L$ and $g \in N$ one has $h(g v)=g\left(g^{-1} h g\right) v \in g \cdot L$ for all $h \in K$ and $v \in L$. The following fundamental theorem from [22] states that the action of $N$ reduces the analysis of the $K$-invariant sublattices to a finite task.

17 Theorem: There are only finitely many orbits of $K$-invariant lattices in $\mathbb{Q}^{n}$ under the action of the rational normalizer $N_{\mathrm{GL}(n, \mathbb{Q})}(K)$.
It remains to consider the determination of representatives for the $N$-orbits of $K$-invariant lattices. First, note that $N$ is an infinite group acting on an infinite set of lattices. Hence the standard methods from computational group theory as outlined in 3.1 do not apply immediately. We therefore address the problem in two steps. In a first step, we consider the centralizer

$$
\begin{aligned}
C & :=C_{\mathrm{GL}(n, \mathbb{Q})}(K) \\
& =\{X \in \mathrm{GL}(n, \mathbb{Q}) \mid X h=h X \text { for all } h \in K\}
\end{aligned}
$$

of the point group $K$. The centralizer $C$ is a subgroup of $N$ of finite index and can be determined by standard methods from representation theory. Note that under the action of $C$ all $K$-invariant maximal sublattices of $L$ containing $q L$ for a prime $q \nmid|K|$ lie in one orbit with $L$ itself. In order to find representatives for the $N$-orbits of $K$-invariant lattices it thus suffices to consider iterated maximal sublattices of index $p^{a}$ for primes $p$ dividing $|K|$.

Since $C$ is of finite index in $N$, there are only finitely many $C$-orbits of $K$-invariant lattices and $N$ acts on these $C$-orbits. Hence, in a second step, we consider the action of $N$ on the $C$-orbits and reduce them to representatives of the $N$-orbits.

\subsection{The Zassenhaus algorithm}

The Zassenhaus algorithm can be used to determine up to isomorphism all space groups with a given point group $K \leq \mathrm{GL}(n, \mathbb{Z})$. It is perhaps one of the most prominent and best-known methods in this area. We refer to [34] for background and further details.

Let $G$ be a space group with point group $K$ in standard form. Then $G$ depends only on the vector system $\tau$ used to build its standard form. The central aim of the Zassenhaus algorithm is to find a set of vector systems which corresponds to the space groups with point group $K$ up to isomorphism.

18 Definition: Let $K \leq \operatorname{GL}(n, \mathbb{Z})$ finite and define $V:=\mathbb{R}^{n} / \mathbb{Z}^{n}$.

$$
\begin{array}{rlrl}
Z^{1}(K, V):=\quad\{\rho: K \rightarrow V \mid \rho(1)=0, \rho(g h)= \\
& \rho(g)+g \rho(h) \text { for all } g, h \in K\} \\
B^{1}(K, V):= & \left\{\rho \in Z^{1}(K, V) \mid \rho(g)=\right. \\
& g v-v \text { for some } v \in V\} \\
H^{1}(K, V):=\quad Z^{1}(K, V) / B^{1}(K, V)
\end{array}
$$


In the definition of $H^{1}(K, V)$ one considers $Z^{1}(K, V)$ as an additive group with pointwise addition. The elements of $Z^{1}(K, V)$ are called cocycles and the elements of $B^{1}(K, V)$ coboundaries with values in $V$. The group $H^{1}(K, V)$ is the first cohomology group of $K$ with values in $V$.

As noted after the definition of vector systems in Section 2.2, every vector system satisfies a cocycle condition as used in the definition of $Z^{1}(K, V)$. This yields that every vector system $\tau$ induces an element $\bar{\tau}$ of $H^{1}(K, V)$ via $\bar{\tau}(g)=\left(\tau(g)+\mathbb{Z}^{n}\right)+$ $B^{1}(K, V)$.

Let $N=N_{\mathrm{GL}(n, \mathbb{Z})}(K)$ be the integral normalizer of $K$. Then $N$ acts on $Z^{1}(K, V)$ via $\rho^{g}(h):=$ $g \rho\left(g h g^{-1}\right)$ for $\rho \in Z^{1}(K, V)$ and $g \in N$. This action leaves $B^{1}(K, V)$ setwise invariant and thus induces an action on $H^{1}(K, V)$. This yields the following fundamental theorem.

19 Theorem: Let $\tau_{1}, \ldots, \tau_{k}$ be a set of vector systems of $K$ such that $\bar{\tau}_{1}, \ldots, \overline{\tau_{k}}$ is a set of of representatives for the $N$-orbits of elements of $H^{1}(K, V)$. Then the space groups in standard form corresponding to the vector systems $\tau_{1}, \ldots, \tau_{k}$ form a set of isomorphism type representatives of space groups with point group $K$.

The standard method to compute $H^{1}(K, V)$ is called the Zassenhaus algorithm and is described in [34]. This is also the standard method to determine $H^{1}(K, V)$ for arbitrary finite groups $K$ and arbitrary modules $V$ in computational group theory nowadays. We recall its main ideas in the following.

Let $\left\langle g_{1}, \ldots, g_{m} \mid r_{1}, \ldots, r_{s}\right\rangle$ be a finite presentation defining the point group $K$. Suppose that a space group $G$ with point group $K$ is given. If the relations are evaluated on elements of $G$ with linear parts corresponding to $g_{1}, \ldots, g_{m}$, then the linear parts will automatically evaluate to the identity matrix. Thus it remains to consider the translation parts which are required to evaluate to elements of the translation subgroup; that is, to vectors with integral entries.

To determine the elements of $Z^{1}(K, V)$, one regards the components of the translation parts of the generators as indeterminates, and obtains a system of linear congruences modulo $\mathbb{Z}$ for these indeterminates by evaluating the relations on the generators. An efficient method to obtain this system of linear congruences is to apply Fox derivatives to the relations; see for example Section 4.2.1 of [13]. The solutions of the obtained linear congruences form the group $Z^{1}(K, V)$.

It now remains to determine $B^{1}(K, V)$. However, this is straightforward by its definition. Once $Z^{1}(K, V)$ and $B^{1}(K, V)$ are available, we obtain $H^{1}(K, V)$.

To apply Theorem 19 in this setting, we determine the integral normalizer $N$ of $K$ (see Section 4.2 ), then we compute the orbits of $N$ on the finite group $H^{1}(K, V)$ (see Section 3.1) and thus we obtain all space groups with point group $K$ up to isomorphism.

20 Lemma: Every coset of $Z^{1}(K, V) / B^{1}(K, V)$ contains a representative whose image is in $\mathbb{Q}^{n} / \mathbb{Z}^{n}$.

Proof: As the elements of $B^{1}(K, V)$ are just the cocycles obtained from conjugation with translations, adjusting the representative by an element of $B^{1}(K, V)$ is the same as translating the origin of the underlying affine space. By choosing an appropriate origin, we can always adjust $\sigma \in Z^{1}(K, V)$ by an element $\delta \in B^{1}(K, V)$ such that the resulting $\sigma+\delta$ has values in $\mathbb{Q}^{n} / \mathbb{Z}^{n}$ only.

Lemma 20 in particular implies that every space group $G$ has a standard form in which the vector system $\tau$ has rational values only.

\section{Classification hierarchy for crystallographic groups}

Space groups can be described in terms of their translation lattices, their point groups and their vector systems. This leads to various possibilities of grouping space groups together and results in a hierarchy of classification levels. In this section we explain the different classification levels suggested by the IUCr Commission on Crystallographic Nomenclature in [16]. First, we recall the definition of the various classes in this hierarchy.

\section{Definition:}

- Two space groups have the same space-group type if they are conjugate by an affine mapping.

- Two space groups are in the same arithmetic crystal class or arithmetic class, for short, if their point groups are conjugate by an element in $\mathrm{GL}(n, \mathbb{Z})$. 
- Two space groups are in the same geometric crystal class or geometric class, for short, if their point groups are conjugate by an element in $\operatorname{GL}(n, \mathbb{Q})$.

- Two space groups are in the same Bravais class if the Bravais groups of their translation lattices lie in the same arithmetic class.

- Two space groups are in the same Bravais system if the Bravais groups of their translation lattices lie in the same geometric class.

- Two geometric classes are in the same crystal system or point-group system if they intersect the same set of Bravais classes.

- Bravais classes with representatives in the same geometric class and geometric classes with representatives in the same Bravais class are joined into a single crystal family.

Figure 1 yields an overview on the various levels of the classification.

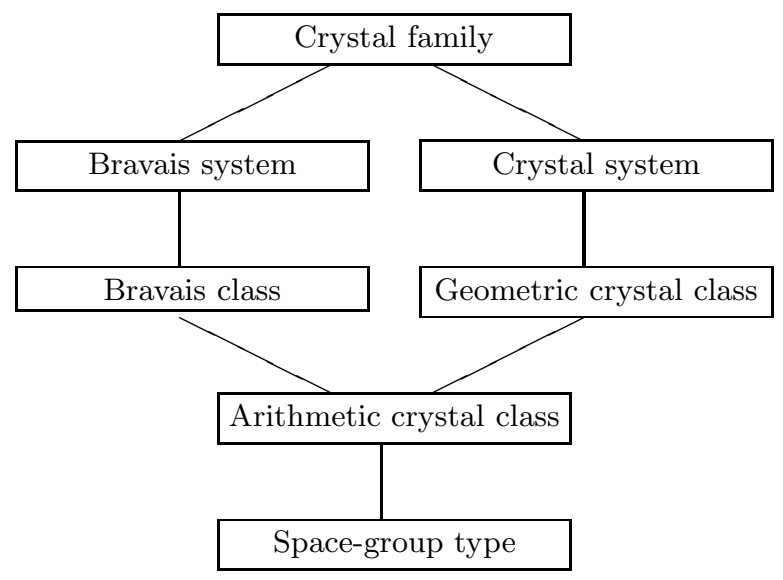

Figure 1: Hierarchy of classification

We discuss these definitions and their background briefly in the following sections.

\subsection{Space-group type}

By the Bieberbach theorems, two space groups $G_{1}$ and $G_{2}$ have the same space-group type if and only if they are isomorphic as abstract groups. Since an isomorphism $\varphi$ has to take the translation subgroup $T_{1}$ of $G_{1}$ to the translation subgroup $T_{2}$ of
$G_{2}$, writing the point groups with respect to lattice bases corresponding to each other via $\varphi$ shows that the point groups of $G_{1}$ and $G_{2}$ can differ by a base change of the translation lattice and thus they are conjugate by an element of $\operatorname{GL}(n, \mathbb{Z})$.

However, there are different possibilities for space groups with given action of the point group on the translation lattice. This is due to the different vector systems. The different possibilities are organized by the first cohomology group $H^{1}\left(K, \mathbb{R}^{n} / \mathbb{Z}^{n}\right)$ as described in Section 4.5 and the different space-group types with point group $K$ are in one-to-one correspondence with the orbits of the integral normalizer $N_{\mathrm{GL}(n, \mathbb{Z})}(k)$ on the vector systems representing $H^{1}\left(K, \mathbb{R}^{n} / \mathbb{Z}^{n}\right)$.

\subsection{Arithmetic crystal class}

Two space groups of the same space-group type lie in the same arithmetic class, as their point groups have be conjugate by an element in $\operatorname{GL}(n, \mathbb{Z})$. The converse is not necessarily true, as the vector systems are not relevant for the arithmetic classes. In effect, the arithmetic classes reflect the interplay of the point groups with the translation lattices.

\subsection{Geometric crystal class}

Two space groups in the same arithmetic class lie in the same geometric class, as $\mathrm{GL}(n, \mathbb{Z}) \leq \mathrm{GL}(n, \mathbb{Q})$ holds. The idea behind this classification level is that the translation lattice of a crystal structure is observed on a microscopic lattice, whereas the macroscopic structure of the crystal allows also transformations of the vector space underlying the translation lattice. Note that the geometric crystal classes were traditionally often just called crystal classes.

\subsection{Bravais class}

The arithmetic and geometric classes collect space groups according to their point groups. Alternatively, space groups may be classified by their translation lattices. It is convenient to characterize a translation lattice by its full group of symmetries; that is, its Bravais group or arithmetic holohedry. Two lattices are said to lie in the same Bravais class if their Bravais groups lie in the same arithmetic class. 
Space groups with translation lattices in the same Bravais class are said to be Bravais equivalent and one extends the notion of Bravais classes to space groups by saying that space groups lie in the same Bravais class if they are Bravais equivalent.

Bravais classes consist of full arithmetic classes by construction and every Bravais class contains a unique arithmetic class of a Bravais group $B$. The other arithmetic classes in the Bravais class are the classes of the subgroups $K$ of $B$ for which the metric tensor of the translation lattice of $B$ is still a generic form in the Bravais manifold $\mathcal{F}(K)$. This is equivalent to saying that the Bravais manifolds $\mathcal{F}(B)$ and $\mathcal{F}(K)$ have the same dimension.

\subsection{Bravais system}

Two Bravais classes of lattices are said to lie in the same Bravais system or lattice system if their Bravais groups lie in the same geometric class. For the lattices this means that one lattice is a sublattice of the other but not isometric to it. Since holohedries are the geometric classes containing a Bravais group, there is a one-to-one correspondence between Bravais systems and holohedries. This notation is extended to space groups by saying that two space groups lie in the same Bravais system if their translation lattices do so.

An equivalent characterization of Bravais systems is often useful and shows more clearly the analogy with the definition of a crystal system as given below. A Bravais system is the union of all Bravais classes intersecting the same set of geometric classes. We say that a Bravais class intersects a geometric class if there is a rational matrix group lying in both the Bravais class and the geometric class.

\subsection{Crystal system}

Two geometric classes are said to lie in the same crystal system if for any representative of the first class there is a representative in the second class such that the representatives have $\operatorname{GL}(n, \mathbb{Q})$ conjugate Bravais groups.

One can see that a crystal system contains at most one holohedry and in low dimensions (up to 4) all crystal systems in fact do contain a holohedry. However, this is not true in general, there are two examples of crystal systems in dimension
5 which do not contain a holohedry and in higher dimensions there is a growing number of such cases.

\subsection{Crystal family}

Note that a crystal family consists of full Bravais systems and of full crystal systems by construction. Consequently, the point groups of two space groups in the same crystal family can be transformed into each other by a finite sequence of the following two operations:

- Take a sub- or supergroup in the same Bravais class.

- Take a conjugate under $\operatorname{GL}(n, \mathbb{Q})$.

Since choosing a different group in the same Bravais class does not change the Bravais manifold, two space groups $G_{1}$ and $G_{2}$ with point groups $K_{1}$ and $K_{2}$ lie in the same crystal family if and only if their Bravais manifolds are transformed into each other by a rational matrix; that is, if there exists $g \in \operatorname{GL}(n, \mathbb{Q})$ such that $\mathcal{F}\left(K_{2}\right)=g^{T} \mathcal{F}\left(K_{1}\right) g$.

\subsection{Enantiomorphism}

So far, we have considered the equivalence of point groups and space groups under abstract transformations. However, crystallography deals with objects in physical space, therefore the operations transforming a structure into an equivalent one have to be orientation-preserving. The distinction between abstract and orientation-preserving transformations gives rise to the notion of enantiomorphism or chirality.

22 Definition: Two groups form an enantiomorphic pair if they are equivalent by an abstract transformation but not by an orientation-preserving transformation. The members of an enantiomorphic pair are said to be enantiomorphic groups.

For example, there are 219 classes of space groups in dimension 3 under abstract affine transformations, but 11 of these classes give rise to enantiomorphic pairs, thus giving the 230 space-group types usually considered in crystallography.

For an elaborate discussion of enantiomorphism on the different levels of the classification hierarchy we refer the reader to [29]. Here, we will only briefly sketch how enantiomorphism on the levels 
of arithmetic classes, geometric classes and spacegroup types can be identified by orbit and stabilizer computations as described in Section 3.1.

We first note that in each of the mentioned cases the equivalence classes are obtained as orbits of a representative $G$ under the action of a group $\mathcal{G}$. For geometric classes we have $\mathcal{G}=\mathrm{GL}(n, \mathbb{Q})$, for arithmetic classes we have $\mathcal{G}=\mathrm{GL}(n, \mathbb{Z})$ and for space-group types we have $\mathcal{G}=\operatorname{Aff}(n)$. We denote the subgroup of orientation-preserving transformations in $\mathcal{G}$ by $\mathcal{G}^{+}$which is a subgroup of index 2 . For $\mathcal{G}=\mathrm{GL}(n, \mathbb{Q})$ and $\mathcal{G}=\mathrm{GL}(n, \mathbb{Z}), \mathcal{G}^{+}$consists of the matrices with positive determinant, for $\mathcal{G}=\operatorname{Aff}(n)$ it consists of the affine mappings with linear part having positive determinant. With this notation, we get the following result.

23 Lemma: The equivalence class of the group $G$ splits under restriction to orientation-preserving transformations into a pair of classes of enantiomorphic groups if and only if the stabilizer $\operatorname{Stab}_{\mathcal{G}}(G)$ of $G$ under $\mathcal{G}$ is contained in $\mathcal{G}^{+}$.

This criterion is readily checked for geometric and arithmetic classes, as the stabilizer $\operatorname{Stab}_{\mathcal{G}}(G)$ in these cases is the rational and integral normalizer $N_{\mathrm{GL}(n, \mathbb{Q})}(G)$ and $N_{\mathrm{GL}(n, \mathbb{Z})}(G)$, respectively. If the normalizer contains no generator of negative determinant, the group is enantiomorphic, otherwise it is not.

The situation is slightly more complicated for the case of space groups. Clearly, the point group of $\operatorname{Stab}_{\mathcal{G}}(G)$ lies in the normalizer $N:=N_{\mathrm{GL}(n, \mathbb{Z})}(K)$ of the point group of $G$. If $N$ only contains elements of positive determinant, then $G$ is enantiomorphic, in fact all space groups with point group $K$ are enantiomorphic in this case. But not every element of $N$ is necessarily the linear part of an element of $\operatorname{Stab}_{\mathcal{G}}(G)$, since also the action on the vector systems has to be considered. Therefore one has to check whether the orbit of the vector system of $G$ under $N$ splits into two orbits, when the action is restricted to the orientation-preserving elements in $N$. If so, $G$ is enantiomorphic, otherwise it is not.

\section{Moving in the classification scheme}

In this section we illustrate how the methods described in Sections 3 and 4 are applied in relation to the classification levels of crystallographic groups. In particular we consider the tasks of

- list all classes on a next lower level into which a given class on a given level splits;

- identify the class of a given space group on a given level;

- to assign a group to a class on a higher level.

\subsection{Listing crystallographic groups}

In this section we consider the task of listing all classes of groups on a certain classification level which belong to the same class on a higher classification level. We will discuss the following steps:

- from arithmetic classes to space-group types;

- from geometric classes to arithmetic classes;

- from Bravais classes to arithmetic classes;

- from crystal families to arithmetic classes.

\section{From arithmetic class to space-group type}

Here the Zassenhaus algorithm is applied. After computing a presentation for the point group $K$, the vector systems are computed and representatives modulo the coboundaries $B^{1}\left(K, \mathbb{R}^{n} / \mathbb{Z}^{n}\right)$ are chosen. Then representatives for the action of the integral normalizer $N_{\mathrm{GL}(n, \mathbb{Z})}(K)$ on the vector systems give the different space-group types with point group in arithmetic class of $K$.

Example: Let

$$
K:=\left\langle g=\left(\begin{array}{cc}
1 & 0 \\
0 & -1
\end{array}\right), \quad h=\left(\begin{array}{cc}
-1 & 0 \\
0 & -1
\end{array}\right)\right\rangle
$$

be the point group $2 \mathrm{~mm}$, then $K$ has the presentation $\left\langle g, h \mid g^{2}=h^{2}=(g h)^{2}=1\right\rangle$. The vector systems for this point group are

$$
t_{g}=\left(\begin{array}{l}
a \\
b
\end{array}\right), t_{h}=\left(\begin{array}{l}
c \\
d
\end{array}\right) \text { with } 2 a \in \mathbb{Z}, 2 b-2 d \in \mathbb{Z}
$$

and the vector systems corresponding to $B^{1}\left(K, \mathbb{R}^{n} / \mathbb{Z}^{n}\right)$ are those with $a=0$ and $b=d$. We therefore find that $H^{1}\left(K, \mathbb{R}^{n} / \mathbb{Z}^{n}\right)$ has four elements and we can choose the vector systems 
representing these elements such that $t_{h}=\left(\begin{array}{l}0 \\ 0\end{array}\right)$ in every case. For $t_{g}$ we get the following choices:

$$
t_{g}^{0}=\left(\begin{array}{c}
0 \\
0
\end{array}\right), t_{g}^{1}=\left(\begin{array}{c}
\frac{1}{2} \\
0
\end{array}\right), t_{g}^{2}=\left(\begin{array}{c}
0 \\
\frac{1}{2}
\end{array}\right), t_{g}^{3}=\left(\begin{array}{c}
\frac{1}{2} \\
\frac{1}{2}
\end{array}\right) .
$$

The normalizer $N_{\mathrm{GL}(n, \mathbb{Z})}(K)$ contains as additional generator the matrix $\left(\begin{array}{ll}0 & 1 \\ 1 & 0\end{array}\right)$ and under the action of this matrix the vector systems with $t_{g}=t_{g}^{1}$ and $t_{g}=t_{g}^{2}$ are interchanged. We therefore obtain three space-group types for the arithmetic class $2 \mathrm{~mm}$.

\section{From geometric class to arithmetic class}

This step is performed via the $K$-invariant sublattices and it is based on the following lemma.

24 Lemma: Let $K \leq \mathrm{GL}(n, \mathbb{Z})$ be a point group. Then the action of $K$ on a $K$-invariant sublattice $L \leq \mathbb{Z}^{n}$ gives a point group in the geometric class of $K$. Conversely, every point group in the geometric class of $K$ is obtained in this way.

Proof: Let $B=\left(b_{1}, \ldots, b_{n}\right)$ be a lattice basis of $L$ and let $X$ be the matrix with columns $b_{i}$. Then the action of $K$ on $L$ with respect to the basis $B$ is given by $X^{-1} K X$ and thus lies in the geometric class of $K$.

Conversely, if $K^{\prime}=X^{-1} K X$ lies in the geometric class of $K$, then $K^{\prime}$ fixes the lattice $X^{-1} \cdot \mathbb{Z}^{n}$, since for $v \in \mathbb{Z}^{n}$ and $g^{\prime}=X^{-1} g X \in K^{\prime}$ one has $g^{\prime}\left(X^{-1} v\right)=X^{-1} g v \in X^{-1} \cdot \mathbb{Z}^{n}$. Multiplying $X^{-1}$ with the least common multiple of the denominators of its entries does not alter $K^{\prime}$ but makes $X^{-1} \cdot \mathbb{Z}^{n}$ a sublattice of $\mathbb{Z}^{n}$

Of course, there are infinitely many $K$-invariant sublattices of $\mathbb{Z}^{n}$, but by Theorem 17 only finitely many of these are relevant in order to split a geometric class into arithmetic classes.

Given a representative $K$ of a geometric class, first the $K$-invariant sublattices are computed up to the action of the normalizer $N_{\mathrm{GL}(n, \mathbb{Z})}(K)$. This yields finitely many sublattices $L_{1}, \ldots, L_{s}$ and the actions $K_{1}, \ldots, K_{s}$ on these sublattices contain a set of representatives for the arithmetic classes in the geometric class of $K$. However, some of the $K_{i}$ may still fall into a single arithmetic class. This is the case if two of the sublattices $L_{i}$ and $L_{j}$ lie in one orbit under the rational normalizer
$N_{\mathrm{GL}(n, \mathbb{Q})}(K)$. We therefore have to test the groups $K_{i}$ on $\mathbb{Z}$-equivalence, thereby getting generators of $N_{\mathrm{GL}(n, \mathbb{Q})}(K)$ as an add-on. Note that computing generators of the rational normalizer directly can only be done by the group automorphism method described in Section 4.2.

Example: (Example of Section 4.4 continued) We have already seen that for $K=31 \mathrm{~m}$ the only $K$-invariant sublattice of $T=\mathbb{Z}^{2}$ which is not contained in $2 T$ or $3 T$ is

$$
L=\left\langle\left(\begin{array}{c}
1 \\
-1
\end{array}\right),\left(\begin{array}{l}
0 \\
3
\end{array}\right)\right\rangle=\left\langle\left(\begin{array}{l}
1 \\
2
\end{array}\right),\left(\begin{array}{l}
-2 \\
-1
\end{array}\right)\right\rangle .
$$

The action of the generators $g, h$ of $K$ with respect to the second basis given for $L$ is

$$
g^{\prime}=\left(\begin{array}{ll}
0 & -1 \\
1 & -1
\end{array}\right), \quad h^{\prime}=\left(\begin{array}{cc}
0 & -1 \\
-1 & 0
\end{array}\right)
$$

and these matrices generate the point group $3 m 1$ representing a different arithmetic class in the geometric class of $31 \mathrm{~m}$.

Since the action of $K$ on $L / 2 L$ is an irreducible $\mathbb{F}_{2} K$-module and primes not dividing $|K|=6$ are irrelevant, the only task remaining is to compute the maximal $\mathbb{F}_{3} K$-invariant submodules of $L / 3 L$. One checks that there is again only one proper submodule and this yields the sublattice $3 T \subset L$, so we are done. We conclude that the geometric class containing $31 \mathrm{~m}$ consists of the arithmetic classes of $31 m$ and $3 m 1$.

\section{From Bravais class to arithmetic class}

Since a Bravais class consists of the subgroups of a Bravais group that have a Bravais manifold of the same dimension, this can be achieved by computing the the lattice of subgroups of the Bravais group. The subgroups are traversed from top to bottom and a branch is cut off if a subgroup has a space of invariant forms of higher dimension. Finally, the obtained subgroups have to be tested for $\mathbb{Z}$-equivalence, but this will only be necessary in very few cases, since most subgroups are distinguished by simple group theoretic invariants.

\section{From crystal family class to arithmetic class}

This is a more complex task which involves both the splitting of geometric classes and of Bravais classes into arithmetic classes. We assume that we 
start with an arbitrary point group $K$ in a crystal family. In a first round we split the geometric class of $K$ into arithmetic classes and compute the Bravais groups for each of these classes. Next, we compute the arithmetic classes in the Bravais classes of these Bravais groups, of course discarding $\mathbb{Z}$ equivalent groups. In a second round, we regard the groups obtained so far as representatives of geometric classes and split again into arithmetic classes and compute Bravais groups. Note that usually only few new Bravais groups will be found, but for these the Bravais classes again have to be split into arithmetic classes. Iterating this process until no new Bravais groups are found yields all arithmetic classes in the crystal family. Note that the computations can be reduced drastically by recording information about $\mathbb{Q}$-equivalence and membership in Bravais classes of the found groups.

\subsection{Identification of crystallographic groups}

This is one of the most common tasks when dealing with crystallographic groups. The general situation is as follows: Given a group on some classification level, find a representative of the class to which the group belongs on this level. Since we can assume that representatives of the classes are known, the task is simplified to the decision, whether two groups on a certain level belong to the same class or not. If the answer is positive, it is desirable that a transformation is explicitly given which transforms the group into the representative.

We will describe the procedures for the different classification levels, the most important being again those for geometric classes, arithmetic classes and space-group types.

\section{Geometric classes}

Assume that we are given two finite matrix groups $K_{1}$ and $K_{2}$ and want to check whether they lie in the same geometric class. One first checks some simple group theoretic invariants like group order, orders and sizes of conjugacy classes and traces of representatives of the conjugacy classes. If the invariants coincide we can embark on one of the following two decision procedures.

As for the integral normalizer one can try to find a conjugating matrix by a direct approach. If the first point group $K_{1}$ is generated by $g_{1}, \ldots, g_{s}$, a conjugating matrix transforms the $g_{i}$ into generators of the second group $K_{2}$. Hence one has to check for all possible generating system $h_{1}, \ldots, h_{s}$ of $K_{2}$ whether the system of linear equations

$$
X g_{i}=h_{i} X \text { for all } 1 \leq i \leq s
$$

has a rational solution matrix $X$. The set of generating systems that have to be tested can be heavily reduced by checking that orders and traces of the generators and their products have to coincide.

A second approach, which is usually faster unless the groups are very small, is to split the geometric class of $K_{1}$ into arithmetic classes and check (by the method described below) whether one of the representatives lies in the same arithmetic class as $K_{2}$.

We note that since we are dealing with finite groups, every representative of a geometric class may be transformed to an integral group. This is done by applying all group elements to the vectors of a lattice basis of $\mathbb{Z}^{n}$ and taking the lattice spanned by the images. Transforming the group to the so found lattice gives an integral matrix group.

\section{Arithmetic classes}

The method based on the computation of the integral normalizer via perfect forms and their directions was already discussed in Section 4.3. Two point groups $K_{1}$ and $K_{2}$ can only lie in the same arithmetic class if their Bravais groups $\mathcal{B}\left(K_{1}\right)$ and $\mathcal{B}\left(K_{2}\right)$ are $\mathbb{Z}$-equivalent. After transforming with the found conjugating matrix for the Bravais groups we may therefore assume that $\mathcal{B}\left(K_{1}\right)=$ $\mathcal{B}\left(K_{2}\right)$ and in that case the groups $K_{1}$ and $K_{2}$ lie in the same arithmetic class if and only if they are conjugate under the integral normalizer of $\mathcal{B}\left(G_{1}\right)$. This is be checked by computing the orbit of $G_{1}$ under $N_{\mathrm{GL}(n, \mathbb{Z})}\left(\mathcal{B}\left(G_{1}\right)\right)$.

\section{Space-group types}

As a first step we have to provide a method to bring a space group to standard form, i.e. such that the translation subgroup is $\mathbb{Z}^{n}$. Since elements in the translation subgroup can be obtained as products of generators with nontrivial linear part, we cannot assume that a lattice basis of the translation subgroup is given explicitly as generators.

In order to compute the translations obtained implicitly from the generators, we require a pre- 
sentation of the point group. We obtain a subgroup of the translation subgroup by evaluating the relations on the space group generators and after adding the translations which are given explicitly as generators the full translation subgroup is obtained as the closure of this subgroup under the action of the point group.

If $n$ linearly independent translations are given which are presumed to generate the translation subgroup, this procedure is simplified to checking that the lattice generated is indeed invariant under the point group and that the relations evaluate to vectors in that lattice.

After having found the translation subgroup $T$, one transforms the space group to a lattice basis of $T$, which yields a space group with point group $K \leq \mathrm{GL}(n, \mathbb{Z})$ and translation subgroup $\mathbb{Z}^{n}$. Finally, a space group in standard form is obtained by reducing the values of the vector system on the generators generators modulo $\mathbb{Z}$

We now consider the problem of deciding whether two space groups $G_{1}$ and $G_{2}$ (in standard form) belong to the same space-group type. For that, we first check whether their point groups $K_{1}$ and $K_{2}$ lie in the same arithmetic class. If not, then the space groups are not equivalent, otherwise we can transform $G_{2}$ such that $K_{2}=K_{1}$. However, we can not identify the generators of $K_{1}$ and $K_{2}$ immediately, since conjugation by an element from the normalizer permutes the point group elements. Instead, we have to compute the orbit of the vector system of $G_{1}$ under the normalizer $N_{\mathrm{GL}(n, \mathbb{Z})}\left(K_{1}\right)$ and check whether for any vector system in the orbit we obtain the vector system of $G_{2}$.

\section{Other levels}

The identification on the higher levels is a combination of the identification methods described above and some enumeration tasks.

- Bravais class: For a given group $K$ we first compute its Bravais group $\mathcal{B}(K)$ and then identify the arithmetic class of $\mathcal{B}(K)$.

- Bravais system: This is the same as for the Bravais class, except that we identify the geometric class of $\mathcal{B}(K)$ instead of the arithmetic class.

- Crystal system: We assume that we have stored for each of the crystal systems which
Bravais classes they intersect. It then remains to split the geometric class of a given group $K$ into arithmetic classes and identify the arithmetic classes of their Bravais groups.

- Crystal family: This is done along the lines of listing the arithmetic classes in a crystal family as described in Section 6.1. If we assume that it is known which Bravais classes are contained in a crystal family, this is reduced to identifying the Bravais class of the given group.

\subsection{Classification of crystallographic groups}

Here, we want to assign a group on a certain classification level to a class on a higher level. In most cases this means to forget some information and to identify the class so obtained on the classification level in question. For example, to assign a spacegroup type to an arithmetic class, the linear part is extracted from the group and it is determined to which arithmetic class the integral matrix group thus obtained belongs.

To assign an arithmetic class to its Bravais class, first its Bravais group is computed and then the class is identified by checking on $\mathbb{Z}$-equivalence.

Even simpler, to assign an arithmetic class to a geometric class, it simply has to be checked to which geometric class the given group belongs. Analogously, on all higher classification levels a given group is just reinterpreted as a representative on the new classification level and has to be identified with one of the representatives on this level.

\section{Computing subgroups}

We discuss the computation of certain subgroups of a given space group $G$. In particular, we consider the problem of computing the maximal subgroups of $G$ and of checking torsion-freeness of $G$. Variations of these methods can be used to determine all subgroups of a given finite index in $G$ and to compute all conjugacy classes of finite subgroups of $G$.

We assume throughout that $G$ is given in standard form. Let $T$ denote the translation subgroup of $G$ and let $K$ be its point group. As a first step, we discuss the theoretic setup which is necessary for such computations. 
25 Lemma: Let $U$ be an arbitrary subgroup of $G$.

(a) $U T=\{u t \mid u \in U, t \in T\}=\langle U, T\rangle$ is a subgroup of $G$ with $T \leq U T \leq G$.

(b) $U \cap T$ is a subgroup of $T$ which is normal in $U T$.

(c) $U /(U \cap T) \cong U T / T$ and $U T / T$ embeds into $K$. The situation is summarized in Figure 2

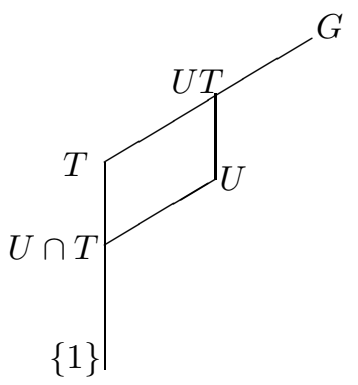

Figure 2: A subgroup $U$ of the space group $G$

Thus every subgroup $U$ of $G$ defines two new subgroups: the subgroup $U T$ and the subgroup $U \cap T$. These play a fundamental role in the computation of subgroups of $G$.

26 Definition: Let $U$ be a subgroup of $G$.

(a) $U$ is called lattice-equal (translationengleich) if $T \leq U$ or, equivalently, if $U \cap T=T$.

(b) $U$ is called class-equal (klassengleich) if $U T=$ G.

The following theorem yields that the latticeequal subgroups of a space group $G$ can be determined by a standard application of methods from computational group theory, see Section 3.

27 Theorem: The lattice-equal subgroups of $G$ correspond one-to-one to the subgroups of the finite point group $K$.

Proof: Follows directly from Lemma 25.

The class-equal subgroups cannot be determined so readily, as there are infinitely many of them. The following theorem shows that they are parameterized by the $K$-invariant sublattices of $T$.

28 Theorem: Let $U$ be a class-equal subgroup of $G$. Then $U \cap T$ is a $K$-invariant sublattice of $T$.

Proof: By Lemma 25 it follows that $U \cap T$ is normal in $U T=G$. Hence $U \cap T$ is $K$-invariant.

\subsection{Maximal subgroups}

The algorithm described in this section has been introduced in [4]. It relies on the following fundamental theorem on subgroups of a space group is known as Hermann's theorem, see [12].

29 Theorem: Let $U \leq G$ be a subgroup of the space group $G$.

(a) There exists a unique group $H$ with $U \leq H \leq$ $G$ such that $H$ is a lattice-equal subgroup of $G$ and $U$ is a class-equal subgroup of $H$.

(b) If $U$ is maximal in $G$, then it is either classequal or lattice-equal.

Proof: (a) By Lemma 25, the group $H=U T$ has the desired properties.

(b) This follows directly from (a): if $U$ is neither class-equal nor lattice-equal, then $U T$ is a proper subgroup of $G$ with $U<U T<G$ and this is not possible for a maximal subgroup.

We split the computation of the maximal subgroups of $G$ in two steps: we first determine the lattice-equal maximal subgroups of $G$ and then we consider the class-equal maximal subgroups of $G$.

The lattice-equal maximal subgroups can be read off from the maximal subgroups of the point group $K$ by Theorem 27 and thus they can be determined readily with standard computational group theory methods. In particular, there are always only finitely many lattice-equal maximal subgroups in a given space group $G$.

It remains to consider the class-equal maximal subgroups. Here the situation is slightly more complicated, as there are always infinitely many classequal maximal subgroups in a given space group $G$. The following follows directly from Theorem 28 .

30 Lemma: Let $U$ be a class-equal maximal subgroup of $G$ and let $L=U \cap T$.

(a) $L$ is a maximal $K$-invariant lattice in $T$.

(b) $U / L$ is a complement to $T / L$ in $G / L$.

(c) $[G: U]=[T: L]$ is a prime power.

Let $L$ be a given maximal $K$-invariant lattice in $T$. Then the (finitely many) maximal subgroups $U$ of $G$ with $U \cap T=L$ can be determined readily by computing all complements to $T / L$ in $G / L$. As 
$G / L$ is a finite group, this can be done with standard methods from computational group theory.

Example: Let $G$ be the 3-dimensional space group with parameters $(4,2,1,1)$, see [2]. Then $G$ is a symmorphic space group whose point group is isomorphic to the cyclic group of order 4 . This yields directly that $G$ has exactly one lattice-equal maximal subgroup, as $C_{4}$ has exactly one maximal subgroup.

We consider the class-equal maximal subgroups of 2-power index in $G$. Let $L$ be a maximal $K$ invariant sublattice of $T$ of 2 -power index in $T$. By Section 4.4 , the sublattice $L$ corresponds to a maximal $K$-submodule of $T / 2 T$. Using the Meataxe, we can readily observe that there are 3 different maximal $K$-submodules in $T / 2 T$ and thus there are 3 different $K$-invariant lattices in $T$ of 2 -power index. For each of these 3 lattices $L$ we observe that $G / L$ is a direct product $G / L \cong T / L \times K \cong C_{2} \times C_{4}$. Hence every of these lattices admits 2 complements to $T / L$ in $G / L$ and we obtain 6 class-equal maximal subgroups of 2-power index in $G$.

We consider the class-equal maximal subgroups of 3-power index in $G$. The point group $K$ of $G$ admits 2 different $K$-invariant lattices in $T$ of 3 power index: one of index 3 and one of index 9 in $T$. Each of these lattices admits 1 conjugacy class of complement to $T / L$ in $G / L$ and both classes are non-trivial. Hence we obtain 12 class-equal maximal subgroups of 3-power index in $G$.

Finally, we note the following corollary of the discussion above.

31 Corollary: Every maximal subgroup $U$ of $G$ is a space group of finite index in $G$.

\subsection{Checking torsion-freeness}

The torsion free space groups are also called Bieberbach groups. They play an interesting role in topology in the study of certain Riemannian manifolds, see for example [3]. Here we consider the problem of checking whether a given space group is torsion free. It turns out that there exists a very efficient method for this purpose.

32 Lemma: If $G$ contains a non-trivial finite subgroup, then it also contains a subgroup of prime order $p$ for some prime $p|| K \mid$.
We denote the projection of a subgroup $U \leq G$ into $K=G / T$ by $\bar{U}$ and the corresponding projection of an element $u \in U$ by $\bar{u}$.

33 Lemma: Let $C$ be a subgroup of prime order $p$ in $G$. Then $C$ is a complement to $T$ in $C T$ and $\overline{C T}$ is a subgroup of order $p$ in $K$.

Hence the approach for checking torsion-freeness is as follows:

(1) Determine the subgroups $H_{1}, \ldots, H_{r}$ of prime order in $K$.

(2) For every $i \in\{1, \ldots, r\}$ :

(a) Let $U_{i} \leq G$ with $T \leq U_{i}$ and $\overline{U_{i}}=H_{i}$.

(b) Check whether $U_{i}$ contains a complement $C_{i}$ to $T$.

(c) If $C_{i}$ exists, then return false.

(3) Return true.

Step (1) is a standard application of methods for finite matrix groups in Gap. We note that it would be sufficient to determine the groups up to conjugacy, since conjugate subgroups behave in the same way in the subsequent algorithm. Step (2a) is straightforward from the definition of $G$.

It remains to consider Step (3). Let $U$ be a subgroup of $G$ with $T \leq U$ and $[U: T]=p$ prime. Then there exists an element $u \in U$ with $U=\langle u, T\rangle$. The aim is to decide whether there exists an $h \in U$ with $h^{p}=1$. A test for this purpose is provided by the following lemma.

34 Lemma: Let $U=\langle u, T\rangle$ with $[U: T]=p$ and let $u=\{g \mid t\}$.

(a) Then $u^{p}=\{1, s\} \in T$ for some $s \in \mathbb{Z}^{n}$.

(b) The group $U$ contains torsion if and only if $-s \in \operatorname{Im}\left(g^{(p-1)}+\ldots+g+\mathbf{1}\right)$.

Proof: (a) As $[U: T]=p$, it follows that $v^{p} \in T$ for every $v \in U$.

(b) The group $U$ contains torsion if and only if $U$ contains an element $h$ of order $p$. We first investigate the possible elements of order $p$ in $U$.

Every element of $U$ is of the form $u^{e} x$ for some $e \in\{0, \ldots, p-1\}$ and $x \in T$. If $h=u^{e} x$ has order $p$, then $e \neq 0$, as otherwise $h=x \in T$. Thus there exists $f \in\{1, \ldots, p-1\}$ such that $e f \equiv 1 \bmod p$, since $p$ is a prime. It follows that $h^{f}=u^{e f} y=u z$ for some $y, z \in T$ and $h^{f}$ has order $p$ also. In summary, it follows that $U$ contains torsion if and 
only if $U$ contains an element $h=u x$ for some $x \in T$ and $h$ has order $p$.

Now let $h \in U$ with $h=u x$ for some $x \in T$. Then

$$
\begin{aligned}
h^{p} & =u x u x \cdots u x \\
& =u^{p} x^{u^{p-1}} x^{u^{p-2}} \cdots x^{u} x \\
& =u^{p} x^{\left(u^{p-1}+u^{p-2}+u+1\right)}
\end{aligned}
$$

Thus $h^{p}=1$ holds if and only if $\left(u^{p}\right)^{-1}=$ $x^{u^{p-1}+u^{p-2}+\ldots+u+1}$ for some $x \in T$. In turn, this translates to $-s \in \operatorname{Im}\left(g^{p-1}+g^{p-2}+\ldots+g+\mathbf{1}\right)$.

\subsection{Variations}

There is a variety of other algorithms available for computing subgroups of various types in a space group. For example:

- A modification (or an iterated application) of the maximal subgroup algorithm allows to determine the subgroups of a given index $l$ in a space group.

- An extension of the algorithm for checking torsion freeness can be used to compute all conjugacy classes of finite subgroups of a space group G.

The idea of the method is as follows. First, we determine all conjugacy classes of subgroups of the point group $K$ of $G$. For every representative $U$ of such a conjugacy class we compute a finite presentation. Then we use the finite presentation to calculate all conjugacy classes of complements to $T$ in $H$ where $H \leq G$ with $T \leq H$ and $H / T=U$. The complement computation is in effect an extension of the method used for checking torsion freeness and reduces to solving a system of linear equations. We refer to [14] for further information.

We note that this algorithm is particularly efficient if the space group $G$ is polycyclic. In this case finite presentations for $K$ and all of its subgroups can be computed readily. Also listing all subgroups of $K$ up to conjugacy is particularly efficient in this case. Again, we refer to [14] for further details.

\section{Computing supergroups}

This section contains a short discussion of the computation of supergroups of a given space group $G$. We restrict this discussion to the determination of minimal supergroups, as an arbitrary supergroup can be constructed by an iterated application of the minimal supergroups method.

The construction of all minimal supergroups of a given space group $G$ is dual to the maximal subgroup computation: if $H$ is a minimal supergroup of $G$, then $G$ is a maximal subgroup of $H$. This suggests the following definition.

35 Definition: Let $H$ be a minimal supergroup of the space group $G$.

(a) $H$ is called lattice-equal, if $G$ is a lattice-equal maximal subgroup of $H$.

(b) $H$ is called class-equal, if $G$ is a class-equal maximal subgroup of $H$.

\subsection{The lattice-equal case}

We consider the computation of the lattice-equal minimal supergroups of a given space group $G$.

First, we analyze the situation. Let $H$ be a lattice-equal minimal supergroup of $G$ and let $T$ denote the translation lattice of $H$. Then $G \cap T=T$ and thus $T$ is also the translation lattice of $G$.

Let $K$ and $K_{H}$ be the point groups of $G$ and $H$, respectively. Then $K_{H}$ is a minimal supergroup of $K$ in $G L(n, \mathbb{Z})$. As a first step, we determine all possible choices for $K_{H}$.

Let $B$ be the Bravais group of $K$. Then $K \leq B$ holds and for the Bravais manifolds we only obtain $\mathcal{F}(B) \subseteq \mathcal{F}(K)$. If $\mathcal{F}(B)=\mathcal{F}(K)$ holds, then it follows that $K_{H}$ embeds into $B$ with $K \leq K_{H} \leq B$ and thus there are only finitely many possibilities for $K_{H}$. However, if $\mathcal{F}(B) \neq \mathcal{F}(K)$, then infinitely many choices for $K_{H}$ are possible as the following example demonstrates.

Example: Let $G$ be the symmorphic space group with point group

$$
K=\left\langle\left(\begin{array}{cc}
-1 & 0 \\
0 & -1
\end{array}\right)\right\rangle
$$

Then for each $a \in \mathbb{Z}$ the symmorphic space group $H_{a}$ with point group

$$
K_{a}=\left\langle\left(\begin{array}{cc}
-1 & 0 \\
0 & -1
\end{array}\right)\left(\begin{array}{cc}
1 & 0 \\
a & -1
\end{array}\right)\right\rangle
$$


is a lattice-equal minimal supergroup of $G$.

The integral normalizer $N=N_{G L(n, \mathbb{Z})}(K)$ acts on the possible point groups $K_{H}$ of the minimal supergroups of $G$. The possible point groups $K_{H}$ fall into finitely many orbits under this action. In most cases, one is only interested in representatives of these orbits so that we can assume that there are only finitely many possible choices for $K_{H}$ in all cases.

To determine a finite set of representatives for the minimal supergroups $U$ of $K$ in $G L(n, \mathbb{Z})$ we use the following ideas. The group $U$ is contained in some Bravais group $B$. We determine those Bravais groups which contain subgroups that are $\mathbb{Z}$ equivalent with $K$. This can be done using standard methods from computational group theory in combination with the $\mathbb{Z}$-equivalence test described in Section 4.3. We then determine all minimal supergroups of $K$ in every of these Bravais groups.

Now suppose that a minimal supergroup $U$ of $K$ in $\mathrm{GL}(n, \mathbb{Z})$ is given. It remains to determine all minimal supergroups $H$ of $G$ with point group $U$. Every such group $H$ is an extension of $T$ by $U$ and the Zassenhaus algorithm can be used to list all such groups up to isomorphism. This algorithm determines in effect a set of vector systems defining the desired groups. Here, we are only interested in extensions of $T$ by $U$ which extend the given extension $G$. This can be computed by a variation of the Zassenhaus algorithm where only those vector systems are determined which extend the vector system defining $G$ as an extension of $T$ by $K$.

\subsection{The class-equal case}

We consider the computation of the class-equal minimal supergroups of a given space group $G$.

First, we analyze the situation. Let $H$ be a classequal minimal supergroup of $G$ and let $T_{H}$ and $T$ denote the translation lattices of $H$ and $G$, respectively. Then $T<T_{H}$ and $K \cong G / T \cong H / T_{H} \cong$ $K_{H}$ if $K$ and $K_{H}$ are the point groups of $G$ and $H$, respectively.

36 Lemma: It follows that $T_{H}$ is a $K$-invariant minimal superlattice of $T$.

Proof: By construction, we obtain that $H=$ $T_{H} G$ and thus $T_{H}$ is invariant under the action of $G$ and hence under the action of $K$.
The minimal superlattices of a given finite subgroup $K$ of $\mathrm{GL}(n, \mathbb{Z})$ can be constructed by an approach dual to the computation of the maximal sublattices as discussed in Section 4.4. We recall the fundamental lemma for this purpose in the following.

37 Lemma: Let $L$ be a minimal superlattice of a lattice $T$. Then $T$ has $p$-power index in $L$ for some prime $p$ and $T \leq L \leq \frac{1}{p} T$.

Thus the minimal superlattices for a given lattice $T$ can be determined as the minimal submodules of the $\mathbb{F}_{p} K$-module $\frac{1}{p} T / T$. In turn, this is a standard application of the Meataxe.

If a minimal superlattice $L$ for $T$ is given, then the minimal supergroup $H$ determined by $L$ can be obtained as $H=L G$.

\section{$9 \quad$ Wyckoff positions}

The Wyckoff positions of a 3-dimensional space group provide a systematic description of the positions of the atoms in an underlying crystal. These Wyckoff positions have first been determined by Niggli in 1919 (see [21]) and later again by Wyckoff (see [32]). They are described in the International Tables for Crystallography [10].

Here we describe an effective algorithm for computing the Wyckoff positions for an arbitrary $n$ dimensional space group. The algorithm is practical and it can be used to determine Wyckoff positions of space groups of much higher dimensions than 3. It has first been described in [4].

First, we recall the theoretical setup for Wyckhoff positions. Let $G$ be an $n$-dimensional space group in standard form. Let $T$ denote its translation lattice, let $K$ be its point group and let $\tau: K \rightarrow \mathbb{R}^{n}: g \mapsto t_{g}$ be its vector system. We assume that $t_{g} \in \mathbb{Q}^{n}$ holds by choosing a suitable origin. Then the elements of $G$ have the form $\left\{k \mid t_{k}+t\right\}$ for $k \in K$ and $t \in \mathbb{Z}^{n}$ and $G$ acts on $V=\mathbb{R}^{n}$ via

$$
\left\{k \mid t_{k}+t\right\}(v)=k v+t_{k}+t
$$

The stabilizer of $v \in V$ under this action is defined as $\operatorname{Stab}_{G}(v)=\{g \in G \mid g(v)=v\}$.

38 Lemma: $\operatorname{Stab}_{G}(v)$ is a finite subgroup of $G$ for every $v \in V$. 
Proof: Let $U=\operatorname{Stab}_{G}(v)$ and suppose that $U$ is infinite. Then $U \cap T$ is non-trivial. Thus there exists an element $u \in U \cap T$ with $u \neq 1$. Then $u=\{1 \mid t\}$ and $u(v)=v+t$. As $u(v)=v$ holds, it follows that $t=0$ and thus a contradiction.

The stabilizer of points in $V$ are used to introduce an equivalence relation on $V$ as in the following definition. Recall that $\operatorname{Stab}_{G}(v)$ is conjugate to $\operatorname{Stab}_{G}(w)$ if there exists a $g \in G$ with $\operatorname{Stab}_{G}(g(v))=\operatorname{Stab}_{G}(w)$ and $\operatorname{Stab}_{G}(g(v))=$ $g \operatorname{Stab}_{G}(v) g^{-1}$.

39 Definition: Let $v, w \in V$. Then $v$ is equivalent to $w$ if $\operatorname{Stab}_{G}(v)$ is conjugate to $\operatorname{Stab}_{G}(w)$ in $G$. We write $v \sim w$ in this case.

It is straightforward to observe that $\sim$ is an equivalence relation on $V$.

40 Definition: The equivalence classes of $\sim$ are called the Wyckoff positions of $G$. They are of the form $W(v)=\{w \in V \mid v \sim w\}$ for $v \in V$ and are full orbits under the action of $G$.

41 Remark: Let $U=\operatorname{Stab}_{G}(v)$ and let $U^{G}=$ $\left\{g U g^{-1} \mid g \in G\right\}$ the conjugacy class of $U$ in $G$. Then $W(v)$ can also be written as $W(v)=\{w \in$ $\left.V \mid \operatorname{Stab}_{G}(y) \in U^{G}\right\}$ and thus $W(v)$ depends on $U^{G}$ only. Hence we also write $W\left(U^{G}\right)$ for $W(v)$.

This also proves that there are only finitely many different Wyckoff positions for a space group $G$, as there are only finitely many conjugacy classes of finite subgroups in $G$. We exhibit the structure of a Wyckoff position in the following.

42 Lemma: Let $U$ be a finite subgroup of $G$.

(a) The fixed points $F(U)=\{v \in V \mid g(v)=$ $v$ for all $g \in U\}$ form an affine space in $V$; that is, they are of the form $F(U)=W+w$ for some $W \leq V$ and $w \in V$ or $F(U)=\emptyset$.

(b) Let $W(U)=\left\{v \in V \mid \operatorname{Stab}_{G}(v)=U\right\}$ the points with stabilizer $U$. Then $W(U) \subseteq F(U)$. Additionally, if $W(U) \neq \emptyset$, then $W(U)$ is dense in $F(U)$.

(c) If $v \in V$ and $U=\operatorname{Stab}_{G}(v)$, then

$$
\begin{aligned}
W(v) & =W\left(U^{G}\right)=\bigcup_{g \in G} W\left(g U g^{-1}\right) \\
& =\bigcup_{g \in G} g(W(U))
\end{aligned}
$$

Proof: (a) If $U=\left\langle g_{1}, \ldots, g_{r}\right\rangle$ and $g_{i}=\left\{m_{i} \mid t_{i}\right\}$ for some $t_{i} \in \mathbb{Q}^{n}$, then $F(U)$ corresponds to the solutions the system of inhomogeneous linear equation $\left(m_{i}-1\right) v=t_{i}$ for $1 \leq i \leq r$ over $\mathbb{Q}$.

(b) $W(U) \subseteq F(U)$ follows directly from the definitions. Further, for every $v \in F(U)$ it follows that $\operatorname{Stab}_{G}(v) \geq U$. Thus $F(U)=W(U) \cup_{H>U} W(H)$ and there are only finitely many possibilities for $H$, since $H$ is a finite subgroup of $G$. If $W(U) \neq \emptyset$, then it follows that $\operatorname{dim}(F(H))<\operatorname{dim}(F(U))$ for every $H>U$, since $F(H) \leq F(U)$ and $F(H) \neq$ $F(U)$. Thus $W(U)=F(U) \backslash \cup_{H>U} W(H)$ is dense in $F(U)$.

(c) The first two equations follow from the definitions. The last equation follows as $g \operatorname{Stab}_{G}(v) g^{-1}=$ $\operatorname{Stab}_{G}(g(v))$ and thus $g(W(U))=W\left(g U g^{-1}\right)$.

Thus we can write a Wyckoff position as an (infinite) union of sets of the form $g(W(U))$ and the basic underlying set $W(U)$ is contained in an affine subspace $F(U)$ of $V$. It is customary to describe the Wyckoff position $W(v)$ by describing the affine space $F(U)$ instead.

\subsection{A first algorithm}

The conjugacy classes of finite subgroups of $G$ can be determined algorithmically, see Section 7.3. For each such conjugacy class $U^{G}$ one can determine $F(U)$ by solving a system of inhomogeneous linear equations over $\mathbb{Q}$ as in the proof of Lemma 42 . It remains to find those conjugacy classes of finite subgroups $U^{G}$ for which $W(U) \neq \emptyset$. This is facilitated by the following lemma. We write $U<_{f} H$ if $U \leq H$ and $U$ has a finite, non-trivial index in $H$.

43 Lemma: Let $U$ be a finite subgroup of G. Then $W(U)=\emptyset$ if and only if $F(U)=$ $\cup_{U<{ }_{f} H} F(H)$.

Proof: First, note that $\cup_{U<{ }_{f} H} F(H) \leq F(U)$ for every $U$. Thus it follows

$$
\begin{aligned}
& W(U)=\emptyset \\
\Leftrightarrow \quad & \operatorname{Stab}_{G}(v)>U \text { for all } v \in F(U) \\
\Leftrightarrow & \exists H>_{f} U \text { with } v \in F(H) \text { for all } v \in F(U) \\
\Leftrightarrow & F(U)=\cup_{U<_{f} H} F(H)
\end{aligned}
$$

This yields the desired result.

This yields a first algorithm for computing all Wyckoff positions of $G$. An improved version of 
this idea has been used in [7]. This method is very effective if the considered group $G$ contains only very few finite subgroups. However, it may happen that a space group contains comparatively many finite subgroups and only a few of them turn up as stabilizers of points. Then this first algorithm incorporates a lot of redundancy and we proceed to suggest a second algorithm which is often more effective.

\subsection{A second algorithm}

Let $H$ be a subgroup of $G$ with $T \leq H \leq G$. In [4] there is an algorithm suggested which computes the fixed point sets $F(U)$ for all $U \leq G$ with $U T=H$. We recall this method here briefly and we show how this can be applied to determine the Wyckoff positions of $G$.

\section{Definition:}

(a) An element $v \in V$ is fixed by $H \bmod T$ if for all $g \in H$ there exists an $h_{g} \in T$ with $g(v)=$ $h_{g}(v)$.

(b) An affine space $A \subseteq V$ is fixed by $H \bmod T$ if for all $v \in A$ and for all $g \in H$ there exists an $h_{g} \in T$ with $g(v)=h_{g}(v)$.

The following lemma yields a computationally useful criterion for the points which are fixed by a subgroup $H \bmod T$.

45 Lemma: Let $H=\left\langle g_{1}, \ldots, g_{r}, T\right\rangle$ and write $g_{i}=\left\{k_{i} \mid t_{i}\right\}$ for $1 \leq i \leq r$ for certain $k_{i} \in K$ and certain $t_{i} \in \mathbb{Q}^{n}$. Then $v \in V$ is fixed by $H \bmod T$ if and only if

$$
\left(k_{i}-1\right) \cdot v \equiv-t_{i} \bmod \mathbb{Z} \text { for } 1 \leq i \leq r .
$$

Proof: The point $v \in V$ is fixed by $H \bmod T$ if and only if there exist $h_{g_{1}}, \ldots, h_{g_{r}} \in T$ with $g_{i}(v)=$ $h_{g_{i}}(v)$ for $1 \leq i \leq r$. Let $h_{g_{i}}=\left\{1, s_{i}\right\}$ for certain $s_{i} \in \mathbb{Z}^{n}$. Then

$$
\begin{aligned}
g_{i}(v)=h_{g_{i}}(v) & \Leftrightarrow k_{i} v+t_{i}=v+s_{i} \\
& \Leftrightarrow \quad\left(k_{i}-1\right) \cdot v=s_{i}-t_{i} \\
& \Leftrightarrow \quad\left(k_{i}-1\right) \cdot v \equiv-t_{i} \bmod \mathbb{Z} .
\end{aligned}
$$

This completes the proof.

This yields that the points fixed by $H \bmod T$ can be computed as the solutions of a system of inhomogeneous linear equations over $\mathbb{Q} / \mathbb{Z}$. This system has the form

$$
M x=b \bmod \mathbb{Z}
$$

where $M \in \mathbb{Z}^{n \times r n}$ and $b \in \mathbb{Q}^{r n}$. To solve such a system, we use a Smith normal form algorithm to compute a diagonal matrix $D$ and invertible integer matrices $P$ and $Q$ with $M=P D Q$. Then

$$
\begin{aligned}
M x=b \bmod \mathbb{Z} & \Leftrightarrow P D Q x=b \bmod \mathbb{Z} \\
& \Leftrightarrow D(Q x)=P^{-1} b \bmod \mathbb{Z} \\
& \Leftrightarrow D x^{\prime}=b^{\prime} \bmod \mathbb{Z}
\end{aligned}
$$

where $D$ is a diagonal integer matrix and $b^{\prime}$ is a rational vector. We denote the diagonal entries of $D$ by $d_{1}, \ldots, d_{n}$.

\section{Lemma:}

(a) The solutions of $M x=b \bmod \mathbb{Z}$ are given by $\left\{v \in V \mid v=Q^{-1} v^{\prime}\right.$ and $\left.D v^{\prime}=b^{\prime}\right\}$.

(b) The solutions of $D x^{\prime}=b^{\prime} \bmod \mathbb{Z}$ are given by those $v^{\prime}=\left(v_{1}^{\prime}, \ldots, v_{d}^{\prime}\right) \in V$ with

$$
v_{i}^{\prime} \in \begin{cases}\left\{\frac{b_{i}^{\prime}+j}{d_{i}} \mid j \in \mathbb{Z}\right\} & \text { if } d_{i} \neq 0 \\ \mathbb{Q} & \text { if } d_{i}=0\end{cases}
$$

Hence the solutions of $M v=b \bmod \mathbb{Z}$ form a union of affine spaces in $V$ which are all of the form $A_{w}=W+w$ with $W \leq V$ such that $\operatorname{dim}(W)=$ $\left|\left\{i \mid d_{i}=0\right\}\right|$ and for some $w \in V$. There are only finitely many choices for $w$ necessary to describe all solutions, since in the case $d_{i} \neq 0$ of Lemma 46b) it is sufficient to consider those $v_{i}^{\prime}$ with $0 \leq j<d_{i}$, as we are only interested in solutions $\bmod \mathbb{Z}$.

Next, we show that the fixed point spaces $F(U)$ for the finite subgroups $U$ of $G$ with $T U=H$ are among these affine spaces fixed by $H \bmod T$.

47 Lemma: Let $U \leq G$ be finite with $T U=H$. Then $F(U)$ is an affine space which is fixed by $H$ $\bmod T$.

Proof: Every element $h \in H$ can be written uniquely in the form $h=k g$ for some $g \in U$ and $k \in T$. Thus for every $v \in F(U)$ it follows that $h(v)=k(g(v))=k(v)$ and thus $F(U)$ is fixed by $H \bmod T$.

Now it remains now to find a set of affine spaces fixed by $H \bmod T$ which yields exactly the fixed point spaces of a set of conjugacy class representatives for the finite subgroups $U$ of $G$ with $T U=H$. 
48 Lemma: Let $A \neq \emptyset$ be a maximal affine space fixed by $H \bmod T$. Then there exists a finite $U \leq G$ with $T U=H$ and $A=F(U)$.

Proof: For every $g \in H$ there exists an $h_{g} \in T$ with $g(v)=h_{g}(v)$ for all $v \in A$. Let $U=\left\{h_{g}^{-1} g \mid\right.$ $g \in H\}$. Then it follows that $T U=H$ by construction. Let $v \in A$. Then $h_{g}^{-1}(g(v))=h_{g}^{-1}\left(h_{g}(v)\right)=v$ and thus $U \leq \operatorname{Stab}_{G}(v)$. Hence $U$ is finite and $A \subseteq F(U)$. As $F(U)$ is an affine space fixed by $H \bmod T$ and $A$ is maximal with this property it follows that $F(U)=A$.

This yields the following algorithm to compute the Wyckoff positions of $G$ :

(1) Determine representatives $\bar{H}_{1}, \ldots, \bar{H}_{k}$ for the conjugacy classes of subgroups of $K$.

(2) Initialize $\mathcal{C}=\emptyset$

(3) For $i \in\{1, \ldots, k\}$ do:

(4) Compute the preimage $H$ of $\bar{H}_{i}$ in $G$.

(5) Compute representatives $A_{1}, \ldots, A_{l}$ for the $T$-orbits of the affine subspaces fixed by $H$ $\bmod T$.

(6) Determine representatives $B_{1}, \ldots, B_{h}$ for the $G / T$-orbits of size $[G: H]$ in the set $\left\{A_{1}, \ldots, A_{l}\right\}$.

(7) Add $B_{1}, \ldots, B_{h}$ to $\mathcal{C}$.

(8) Return $\mathcal{C}$.

\subsection{An example}

Let $G$ be the space group of dimension 2 with $G=$ $\left\langle a, t_{1}, t_{2}\right\rangle$ where

$$
\begin{gathered}
a=\left(\begin{array}{rrr}
0 & 1 & 0 \\
-1 & 0 & 0 \\
0 & 0 & 1
\end{array}\right) \text { and } \\
t_{1}=\left(\begin{array}{lll}
1 & 0 & 1 \\
0 & 1 & 0 \\
0 & 0 & 1
\end{array}\right), t_{1}=\left(\begin{array}{lll}
1 & 0 & 0 \\
0 & 1 & 1 \\
0 & 0 & 1
\end{array}\right) .
\end{gathered}
$$

Then $G$ is a symmorphic space group in standard form and its point group $K$ is a cyclic group of order 4 . We illustrate the two algorithms introduced above on this example.

\section{The first algorithm}

The space group $G$ has 6 conjugacy classes of finite subgroups. Representatives for these are listed in the following:

$$
\begin{aligned}
U_{1} & =\langle a\rangle \cong C_{4} \\
U_{2} & =\left\langle t_{2} a\right\rangle \cong C_{4} \\
U_{3} & =\left\langle a^{2}\right\rangle \cong C_{2} \\
U_{4} & =\left\langle t_{2} a^{2}\right\rangle \cong C_{2} \\
U_{5} & =\left\langle t_{1} t_{2} a^{2}\right\rangle \cong C_{2} \\
U_{6} & =\{1\}
\end{aligned}
$$

For every of these 6 representatives, we can determine its corresponding fixed point space by solving a linear equation. We illustrate this in detail on the example $U_{2}$.

Recall that $F\left(U_{2}\right)=\left\{v \in \mathbb{R}^{2} \mid\left(t_{2} a\right)(v)=v\right\}$. Let $a=\{k \mid 0\}$ and $t_{i}=\left\{1 \mid s_{i}\right\}$. Then the elements $v \in \mathbb{R}^{2}$ lying in $F\left(U_{2}\right)$ can be determined by solving the equation $(k-1) v=-s_{2}$; that is

$$
\left(\begin{array}{rr}
-1 & 1 \\
-1 & -1
\end{array}\right)\left(\begin{array}{l}
v_{1} \\
v_{2}
\end{array}\right)=\left(\begin{array}{r}
0 \\
-1
\end{array}\right) \text {. }
$$

This yields

$$
v=\left(\begin{array}{c}
\frac{1}{2} \\
\frac{1}{2}
\end{array}\right)
$$

as the only possible solution.

The fixed point spaces for the other finite subgroups can be determined by the same approach. The resulting fixed point spaces are listed in the following.

$$
\begin{aligned}
& F\left(U_{1}\right)=\{0\} \\
& F\left(U_{2}\right)=\left\{\left(\begin{array}{c}
\frac{1}{2} \\
\frac{1}{2}
\end{array}\right)\right\} \\
& F\left(U_{3}\right)=F\left(U_{1}\right) \\
& F\left(U_{4}\right)=\left\{\left(\begin{array}{c}
0 \\
\frac{1}{2}
\end{array}\right)\right\} \\
& F\left(U_{5}\right)=F\left(U_{2}\right) \\
& F\left(U_{6}\right)=\mathbb{R}^{2}
\end{aligned}
$$

Hence the Wyckoff positions of $G$ are described by $F\left(U_{1}\right), F\left(U_{2}\right), F\left(U_{4}\right)$ and $F\left(U_{6}\right)$.

\section{The second algorithm}

The point group $K$ of $G$ has 3 conjugacy classes of subgroups. Let $a=\{k \mid 0\}$ as above and note that $K=\langle k\rangle \cong C_{4}$. Thus representatives for the conjugacy classes of subgroups of $K$ are $K, U=\left\langle k^{2}\right\rangle$ and $\{1\}$. We apply the second algorithm to these 
subgroups for determining the Wyckoff positions of $G$.

Choose $H=G$ as a preimage for the subgroup $K$ of $K$. We want to compute representatives for the $T$-orbits of the affine subspaces fixed by $H \bmod$ $T$. These are the solutions of $(k-1) v \equiv 0 \bmod \mathbb{Z}$; that is,

$$
\left(\begin{array}{rr}
-1 & 1 \\
-1 & -1
\end{array}\right)\left(\begin{array}{l}
v_{1} \\
v_{2}
\end{array}\right) \equiv\left(\begin{array}{l}
0 \\
0
\end{array}\right) \bmod \mathbb{Z} .
$$

Let $M=k-1$. Then $M=P D Q$ for the matrices

$P=\left(\begin{array}{rr}-1 & 0 \\ -1 & -1\end{array}\right), D=\left(\begin{array}{ll}1 & 0 \\ 0 & 2\end{array}\right), Q=\left(\begin{array}{rr}1 & -1 \\ 0 & 1\end{array}\right)$

This yields

$$
\begin{aligned}
& M v \equiv 0 \bmod \mathbb{Z} \\
= & (P D Q) v \equiv 0 \bmod \mathbb{Z} \\
= & D v^{\prime} \equiv 0 \bmod \mathbb{Z} \text { and } v^{\prime}=Q v \\
= & v^{\prime} \in\left\{\left(\begin{array}{c}
0 \\
0
\end{array}\right)\left(\begin{array}{c}
0 \\
\frac{1}{2}
\end{array}\right)\right\} \text { and } v=Q^{-1} v^{\prime} \\
= & v \in\left\{\left(\begin{array}{c}
0 \\
0
\end{array}\right)\left(\begin{array}{c}
\frac{1}{2} \\
\frac{1}{2}
\end{array}\right)\right\}
\end{aligned}
$$

In summary, we obtain two Wyckoff positions from the subgroup $K$ of $K$ and these correspond to $F\left(U_{1}\right)$ and $F\left(U_{2}\right)$ in the first algorithm.

Choose $H=\left\langle a^{2}, t_{1}, t_{2}\right\rangle$ as a preimage for the subgroup $U$ of $K$. We want to compute representatives for the $T$-orbits of the affine subspaces fixed by $H \bmod T$. These are the solutions of $\left(k^{2}-1\right) v \equiv 0 \bmod \mathbb{Z}$; that is,

$$
\left(\begin{array}{rr}
-2 & 0 \\
0 & -2
\end{array}\right)\left(\begin{array}{l}
v_{1} \\
v_{2}
\end{array}\right) \equiv\left(\begin{array}{l}
0 \\
0
\end{array}\right) \bmod \mathbb{Z} .
$$

Solutions for this equation can be read off readily as

$$
v \in\left\{\left(\begin{array}{c}
0 \\
0
\end{array}\right)\left(\begin{array}{c}
0 \\
\frac{1}{2}
\end{array}\right)\left(\begin{array}{c}
\frac{1}{2} \\
0
\end{array}\right)\left(\begin{array}{c}
\frac{1}{2} \\
\frac{1}{2}
\end{array}\right)\right\} .
$$

The first and last of these choices for $v$ are already covered by the Wyckoff positions for the subgroup $K$. Hence we only have to consider the second and third choice for $v$. These two choices lie in one orbit under the action of $G$ and hence we obtain one Wyckoff position from these two choices.

In summary, we obtain one Wyckoff position from the subgroup $U$ of $K$ and this corresponds to $F\left(U_{4}\right)$ in the first algorithm.

Finally, the full space arises as Wyckoff position for the trivial subgroup of $K$.

\section{Generalizations}

In this section we briefly discuss some variations and generalizations of the methods discussed in this paper: we consider their application to subperiodic groups and colour groups.

\subsection{Subperiodic groups}

49 Definition: A subgroup $G$ of $\mathrm{E}(n)$ is a $s u b$ periodic group if its translation subgroup $T=$ $G \cap T(n)$ is a lattice of rank $s<n$ and its point group $K=G / T$ is finite. This is equivalent to saying that $G$ is a subgroup of a space group with a translation subgroup of smaller rank.

For $n=2$ and $s=1$ these groups are known as frieze groups or strip pattern groups, for $n=3$ and $s=1$ these groups are called rod groups and for $n=3$ and $s=2$ they are called layer groups.

For many tasks, subperiodic groups can be handled just as space groups, but we obtain an especially useful standard form for subperiodic groups if we choose the underlying basis appropriately.

50 Definition: A subperiodic group $G$ with translation subgroup $T$ is given in standard form if $G$ is represented with respect to a basis $B=$ $\left(b_{1}, \ldots, b_{n}\right)$ such that $\left(b_{1}, \ldots, b_{s}\right)$ is a lattice basis of $T$ and $\left(b_{s+1}, \ldots, b_{n}\right)$ span a lattice which is orthogonal to $T$ and invariant under the point group $K=G / T$.

For a subperiodic group $G$ in standard form the point group $K$ consists of integral matrices $g$ which are in block diagonal form with blocks of sizes $s \times s$ and $(n-s) \times(n-s)$, respectively:

$$
g=\left(\begin{array}{c|c}
g_{E} & 0 \\
\hline 0 & g_{I}
\end{array}\right) \in \mathrm{GL}(s, \mathbb{Z}) \times \operatorname{GL}(n-s, \mathbb{Z}) .
$$

We denote the components of $g$ by $g_{E}$ for the action on the exterior space $T$ and $g_{I}$ for the action on the interior space $\left\langle b_{s+1}, \ldots, b_{n}\right\rangle$. For the sake of brevity we will denote the block diagonal matrix $g$ by the tuple $\left(g_{E}, g_{I}\right)$.

We note that the vector system $\tau: K \rightarrow \mathbb{R}^{n}:$ $g \mapsto t_{g}$ has only non-zero entries in the first $s$ components corresponding to $T$ and can therefore be thought of as a vector system $\tau^{\prime}: K \rightarrow \mathbb{R}^{s}$. A subperiodic group $G$ with point group $K$ is thus given 
in standard form as

$G=\left\{\left(\begin{array}{c|c|c}g_{E} & 0 & t_{g}+t \\ \hline 0 & g_{I} & 0 \\ \hline 0 & 0 & 1\end{array}\right) \mid\left(g_{E}, g_{I}\right) \in K, t \in \mathbb{Z}^{s}\right\}$.

Most of the algorithms for space groups can either be readily applied to subperiodic groups or be adapted in a straightforward fashion. As examples, we mention the computation of the integral normalizer, the Zassenhaus algorithm and the classification of subperiodic groups.

\section{The integral normalizer}

Let $K \leq \mathrm{GL}(n, \mathbb{Z})$ be the point group of a subperiodic group in standard form and denote by $K_{E}$ en $K_{I}$ the actions of $K$ on $T$ and on the orthogonal complement of $T$, respectively. Then $K$ is a subdirect product of $K_{E}$ and $K_{I}$; that is, a subgroup of the direct product $K_{E} \times K_{I}$ (see Section 2.1 of [13] for a discussion of subdirect products).

It is important to note that the integral normalizer in the situation of subperiodic groups is not the full normalizer in $\operatorname{GL}(n, \mathbb{Z})$, but only the normalizer in $\operatorname{GL}(s, \mathbb{Z}) \times \mathrm{GL}(n-s, \mathbb{Z})$, since the exterior and interior space may not be interchanged. There are now two ways to obtain this normalizer $N:=N_{\mathrm{GL}(s, \mathbb{Z}) \times \mathrm{GL}(n-s, \mathbb{Z})}(K)$ :

(1) Compute the normalizers $N_{E} \quad:=$ $N_{\mathrm{GL}(s, \mathbb{Z})}\left(K_{E}\right)$ and $N_{I}:=N_{\mathrm{GL}(n-s, \mathbb{Z})}\left(K_{I}\right)$ of the two components of $K$, then $N$ is a subgroup of the direct product $N_{E} \times N_{I}$ and can be found via an orbit computation.

(2) Compute the full normalizer $N^{\prime}:=$ $N_{\mathrm{GL}(n, \mathbb{Z})}(K)$ and find $N$ as the subgroup of $N^{\prime}$ stabilizing $T$.

Which of these approaches is preferable depends mainly on the index of $K$ in $K_{E} \times K_{I}$, but both are usually very efficient.

\section{The Zassenhaus algorithm}

In order to find the subperiodic groups with given point group $K$, the Zassenhaus algorithm can be applied almost unaltered. The only change is that only vector systems $\tau: K \rightarrow \mathbb{R}^{n}$ are considered that have zero entries in the last $n-s$ components corresponding to the interior space.
Classifying subperiodic groups

We mentioned that the normalizer of the point group of a subperiodic group may not interchange the exterior and interior spaces. The same issue arises when classifying the point groups into arithmetic and geometric classes. Instead of conjugating with matrices from $\mathrm{GL}(n, \mathbb{Z})$ or $\mathrm{GL}(n, \mathbb{Q})$, one only conjugates with transformations from $\operatorname{GL}(s, \mathbb{Z}) \times$ $\mathrm{GL}(n-s, \mathbb{Z})$ and $\operatorname{GL}(s, \mathbb{Q}) \times \mathrm{GL}(n-s, \mathbb{Q})$, respectively. However, since the basis chosen for the interior space is a lattice basis of an arbitrary lattice invariant under $K$, arithmetic classes for the action on the interior space are of limited use. One therefore defines a mixed form of arithmetic and geometric equivalence for the point groups of subperiodic groups as follows.

51 Definition: Two subperiodic groups $G_{1}, G_{2}$ with translation subgroups $T_{1}, T_{2}$ of rank $s$ and point groups $K_{1}, K_{2}$ are said to lie in the same arithmetic-geometric class if the actions of $K_{1}$ on $T_{1}$ and of $K_{2}$ on $T_{2}$ lie in the same arithmetic class and the actions of $K_{1}$ and $K_{2}$ on the orthogonal complements of $T_{1}$ and $T_{2}$, respectively, lie in the same geometric class.

The arithmetic-geometric class of a point group $K$ is therefore obtained as the orbit of $K$ under $\mathrm{GL}(s, \mathbb{Z}) \times \mathrm{GL}(n-s, \mathbb{Q})$. For a thorough discussion of arithmetic and geometric classes in the context of subperiodic groups, we refer the reader to [17].

\subsection{Colour groups}

So far we have only been concerned with space groups acting on an affine space. However, the points of the affine space may also be endowed with further properties, e.g. a magnetic spin or the type of an atom. One therefore extends the notion of space groups to groups which act on the points of the affine space together with their additional properties. If this additional property can only take one of finitely many values, these values are usually identified with colours and one thus calls these groups colour groups. Of special interest is the case of two colours (representing for example spins up and down) and these groups are known as blackand-white groups. Also the action on several additional properties has been considered, these groups are usually called antisymmetry groups. They can be seen as colour groups where the action on the 
colours is not necessarily transitive, i.e. the colours may fall into several orbits. An overview of the concepts and results obtained for antisymmetry groups is given in [33].

It was already noted by Heesch in [11] that blackand-white groups can be viewed as special subperiodic groups where the action on the interior space is restricted to \pm 1 . This concept can be extended to arbitrary colour groups, where the action on the interior space is the permutation action on the colours. We not that the dimension of the interior space can be chosen as $m-1$ for a group acting on $m$ colours, since the symmetric group on $m$ points has an integral representation of dimension $m-1$.

Since we think of a space group as acting on points in general position, pure colour operations are not allowed, hence the trivial element of the space group only occurs with the trivial permutation on the colours. If we denote the colour group by $\mathcal{G}$, the space group giving the action of $\mathcal{G}$ on the affine space by $G$ and the permutation action of $\mathcal{G}$ on the colours by $P$, we can describe the colour group $\mathcal{G}$ as follows:

52 Lemma: A colour group $\mathcal{G}$ is a subdirect product $\mathcal{G} \leq G \times P$ of a space group $G$ and a permutation group $P$ such that:

(a) The index of $\mathcal{G}$ in $G \times P$ is $|P|$.

(b) The projections of $\mathcal{G}$ to the components of the direct product are surjective.

We briefly describe two different ways to approach colour groups computationally. The first one starts with a space group and a number $m$ of colours. Since we want a transitive action on the colours, the stabilizer of any colour is a subgroup of index $m$ and the stabilizers for the different colours are conjugate subgroups. In order to find the different ways to realize a space group $G$ as a colour group acting on $m$ colours, one therefore has to find the different subgroups of index $m$ in $G$, up to conjugacy by the normalizer. This task can be split into two parts, since the subgroups of index $m$ are found by first taking a lattice-equal subgroup $H$ of index $m_{1}$ in $G$ and then a class-equal subgroup of index $m_{2}$ in $H$ such that $m=m_{1} \cdot m_{2}$.

A second approach uses the above lemma more explicitly. The conditions of the lemma imply that the permutation group $P$ is isomorphic to a factor group of $G$. For a given space group $G$, this restricts the possibilities of actions on the colours heavily, since the permutation group $P$ and the point group $K$ of $G$ need to have a common factor group $K / K_{1} \cong P / P_{1}$ such that $P_{1}$ is an abelian group. The last statement is due to the fact that $P_{1}$ in this situation has to be isomorphic to a factor group of the translation subgroup $T$.

Just as for the subperiodic groups the computational methods for space groups can also be readily adapted to colour groups. As an example we refer to [30] where the 4-dimensional black-and-white groups are enumerated. Again, the main modifications are to adjust the normalizer to the situation considered and to restrict the vector systems to the exterior space.

\section{References}

[1] L. Bieberbach. Über die Bewegungsgruppen der Euklidischen Räume. (Erste Abhandlung). Math. Ann. 70 (1911) 297-336.

[2] H. Brown, R. Bülow, J. Neubüser, H. Wondratschek, and H. Zassenhaus. Crystallographic groups of four-dimensional space. John Wiley, New York, 1978.

[3] L.S. Charlap. Bieberbach Groups and Flat Manifolds. Springer, New York, 1986.

[4] B. Eick, F. Gähler, and W. Nickel. Computing maximal subgroups and Wyckhoff positions of space groups. Acta Cryst. A53, (1997) 467-474.

[5] B. Eick, F. Gähler, and W. Nickel. Cryst computing with crystallographic groups. A refereed Gap 4 package (see [9]), 2001.

[6] V. Felsch and F. Gähler. CrystCat - a library of crystallographic groups. A refereed Gap 4 package (see [9]), 2000.

[7] J. Fuksa and P. Engel. Derivation of Wyckhoff positions of $n$-dimensional space groups. Theoretical considerations. Acta Cryst. A50 (1994) 778-792.

[8] F. Gähler. An interface to Carat (see [25]). A refereed Gap 4 package (see [9]), 2001.

[9] The Gap Group. Gap - Groups, Algorithms and Programming. Version 4.4. Available from http://www.gap-system.org, 2004. 
[10] T. Hahn (ed.). International Table for Crystallography, Vol. A. 5th ed. Kluwer Academic Publishers, Dordrecht, 2002.

[11] H. Heesch. Über die vierdimensionalen Gruppen des dreidimensionalen Raumes. Z. Krist. 73 (1930) 325-345.

[12] C. Hermann. Zur systematischen Strukturtheorie. IV. Untergruppen. Z. Krist. 69 (1929) 533-555.

[13] D.F. Holt and W. Plesken. Perfect Groups. Oxford University Press, Oxford, 1989.

[14] D.F. Holt, B. Eick, and E.A. O'Brien. Handbook of Computational Group Theory. Chapman \& Hall/CRC, Boca Raton, 2005.

[15] D.-O. Jacquet-Chiffelle. Trois théorèms de finitude pour les $G$-formes. J. Théor. Nombres Bordeaux 7 (1995) 165-176.

[16] T. Janssen, J.L. Birman, F. Dénoyer, V.A. Koptsik, J.R. Verger-Gaugry, D. Weigel, A. Yamamoto, S.C. Abrahams, and V. Kopsky. Report of a Subcommittee on the Nomenclature of $n$-Dimensional Crystallography. II. Symbols for arithmetic crystal classes, Bravais classes and space groups. Acta Cryst. A58 (2002) 605-621.

[17] K.-J. Köhler. On the structure and the determination of $n$-dimensional partially periodic crystallographic groups. Comm. Math. Chem. 10 (1981) 27-53.

[18] Computational Algebra Group, School of Mathematics and Statistics, University of Sydney. The Magma Computational Algebra System for Algebra, Number Theory and Geometry. http://magma.maths. usyd. edu. au/magma, 2004.

[19] J. Martinet. Perfect Lattices in Euclidean Spaces. Springer, Berlin, 2002.

[20] J. Neubüser, W. Plesken, and H. Wondratschek. An emendatory discursion on defining crystal systems. Comm. Math. Chem. 10 (1981) 77-96.

[21] P. Niggli. Geometrische Kristallographie des Diskontinuums. Bornträger, Leipzig, 1919.
[22] J. Opgenorth, W. Plesken, and T. Schulz, T. Crystallographic Algorithms and Tables. Acta Cryst. A54 (1998) 517-531.

[23] J. Opgenorth. Dual Cones and the Voronoi Algorithm. Exper. Math. 10 (2001) 599-608.

[24] W. Plesken. Bravais groups in low dimensions. Comm. Math. Chem. 10 (1981) 97-119.

[25] W. Plesken, J. Opgenorth, and T. Schulz. Carat - a package for mathematical crystallography. J. Appl. Cryst. 31 (1998) 827-828. Available from http://wwwb.math.rwth-aachen.de/carat.

[26] W. Plesken and T. Schulz. Counting Crystallographic groups in Low Dimensions. Exper. Math. 9 (2000) 407-411.

[27] W. Plesken and B. Souvignier. Computing isometries of lattices. J. Symb. Comp. 24 (1997) 327-334.

[28] R. Schmied and K.K. Lehmann. Computergenerated character tables and nuclear spin statistical weights: application to benzene dimer and methane dimer. Journal of Molecular Spectroscopy 226 (2004) 201-202.

[29] B. Souvignier. Enantiomorphism of crystallographic groups in higher dimensions with results in dimensions up to 6. Acta Cryst. A59 (2003) 210-220.

[30] B. Souvignier. The four-dimensional magnetic point and space groups. To appear in Z. Krist. (2005).

[31] G.F. Voronoï. Nouvelles applications des paramètres continus à la théorie des formes quadratiques. II: Recherches sur les parallélloèdres primitifs, première partie. J. Reine Angew. Math. 134 (1908) 198-287.

[32] R.W.G. Wyckoff. The Analytical Expression of the Results of the Theory of Space Groups. Carnegie Institute, Washington, 1922.

[33] A.M. Zamorzaev, A.F. Palistrant. Antisymmetry, its generalizations and geometrical applications. Z. Krist. 151 (1980) 231-248.

[34] H. Zassenhaus. Über einen Algorithmus zur Bestimmung der Raumgruppen. Comm. Math. Helv. 21 (1948) 117-141. 